\title{
Single-cell RNA-seq reveals novel regulators of human embryonic stem cell differentiation to definitive endoderm
}

\author{
Li-Fang Chu ${ }^{1 *}{ }^{*}$, Ning Leng ${ }^{1,6 \dagger}$, Jue Zhang ${ }^{1}$, Zhonggang Hou ${ }^{1,7}$, Daniel Mamott ${ }^{1}$, David T. Vereide ${ }^{1}$, Jeea Choi ${ }^{4}$, \\ Christina Kendziorski ${ }^{5}$, Ron Stewart ${ }^{1}$ and James A. Thomson ${ }^{1,2,3^{*}}$
}

\begin{abstract}
Background: Human pluripotent stem cells offer the best available model to study the underlying cellular and molecular mechanisms of human embryonic lineage specification. However, it is not fully understood how individual stem cells exit the pluripotent state and transition towards their respective progenitor states.

Results: Here, we analyze the transcriptomes of human embryonic stem cell-derived lineage-specific progenitors by single-cell RNA-sequencing (scRNA-seq). We identify a definitive endoderm (DE) transcriptomic signature that leads us to pinpoint a critical time window when DE differentiation is enhanced by hypoxia. The molecular mechanisms governing the emergence of DE are further examined by time course scRNA-seq experiments, employing two new statistical tools to identify stage-specific genes over time (SCPattern) and to reconstruct the differentiation trajectory from the pluripotent state through mesendoderm to DE (Wave-Crest). Importantly, presumptive DE cells can be

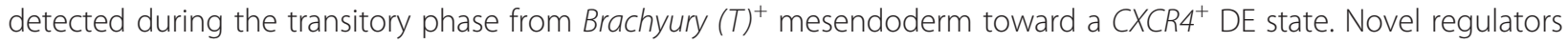
are identified within this time window and are functionally validated on a screening platform with a T-2A-EGFP knock-in reporter engineered by CRISPR/Cas9. Through loss-of-function and gain-of-function experiments, we demonstrate that KLF8 plays a pivotal role modulating mesendoderm to DE differentiation.

Conclusions: We report the analysis of 1776 cells by scRNA-seq covering distinct human embryonic stem cell-derived progenitor states. By reconstructing a differentiation trajectory at single-cell resolution, novel regulators of the mesendoderm transition to DE are elucidated and validated. Our strategy of combining single-cell analysis and genetic approaches can be applied to uncover novel regulators governing cell fate decisions in a variety of systems.
\end{abstract}

Keywords: Single-cell RNA-seq, Embryonic stem cells, Mesendoderm, Brachyury, Definitive endoderm, Wave-Crest, SCPattern, KLF8, CRISPR/Cas9

\section{Background}

The three primary germ layers composed of lineagespecific progenitors are critical for the establishment of the embryonic body plan [1-4]. Directional differentiation protocols have efficiently driven human pluripotent stem cells into progenitor populations mimicking those of the embryonic ectoderm, mesoderm, endoderm, and extraembryonic lineages [5-18]. However, it is not fully understood how individual embryonic stem (ES) cells exit

\footnotetext{
* Correspondence: Ichu@morgridge.org; jthomson@morgridge.org

${ }^{\dagger}$ Equal contributors

${ }^{1}$ Morgridge Institute for Research, Madison, WI, USA

Full list of author information is available at the end of the article
}

the pluripotent state and give rise to lineage-specific progenitors.

Among the three primary germ layers, the definitive endoderm (DE) is the internal layer of the embryonic gut, formed by the recruitment of epiblast cells through the primitive streak. The DE cells give rise to a variety of functional specialized epithelial cell types that line the developing gut tube and contribute to vital organs or tissues such as the lungs, trachea, esophagus, liver, stomach, intestine, thyroid, thymus, and pancreas [1-3]. These endoderm-derived organs support indispensible functions in adults, such as gas exchange in respiration, mechanical and chemical digestion, and blood glucose 
homeostasis and detoxification. Therefore, human pluripotent stem cell-derived DE cells are an instrumental resource for regenerative medicine $[7,9,10,15,18,19]$. However, the factors governing the transition from epiblast-derived precursors to the DE state is not fully understood.

Mesendoderm represents a transient state, composed of a migratory cell population emerging from the primitive streak. Its emergence is accompanied by the activation of the transcription factor Brachyury (T), which marks the onset of gastrulation $[2,11,20]$. As gastrulation continues, mesendoderm contributes to mesoderm or DE. Once the lineage decision is segregated, $T$ expression appears to be continually associated with certain mesodermal derivatives but not DE derivatives [11, 21, 22]. This represents a key developmental juncture when cell fate decisions have been made from a broad multi-potent state (mesendoderm) towards a more restricted state (definitive endoderm). Therefore, we designed our scRNA-seq experiments to detect signals that could promote DE differentiation and then followed up these experiments with a detailed time course to identify the critical time window in which mesendoderm transitions to the DE state.

Standard methods for transcriptome-wide profiling of differentiation involves the collection of thousands to millions of cells for deep sequencing (bulk RNA-seq) at one or several time points. With this approach, cellular heterogeneity cannot be resolved since variably expressed genes will be averaged or - if exclusively expressed in rare cells - completely missed. Single-cell RNA-seq (scRNA-seq), on the other hand, is able to characterize cell-to-cell variation and reveal transcriptomic signatures unique to individual cells [23-25]. Such analyses can provide novel insights into the responses to extrinsic signals and reveal intrinsic factors that control cell fate decisions. These insights can then guide the genesis of more sophisticated differentiation protocols and quality control assays.

To understand the distinctions between DE cells and the other lineage-specific progenitors, we examined their transcriptomes by scRNA-seq. Our analysis revealed a DEspecific signature that is enriched for NODAL and WNT signaling pathways as well as metabolism-related gene expression. The latter category of genes led us to define a time window in which hypoxia could enhance DE marker expression. Based on this observation, we hypothesized that the emergence of nascent DE cells occurs as soon as two days post differentiation from the pluripotent state.

Compared to single time point experiments, time course scRNA-seq has the potential to reveal detailed cell state transitions [26-28]. To pinpoint the exact timing of DE cell emergence, we profiled the transition of single human ES cells to mesendoderm then to the DE state over four days of differentiation. To analyze the transition at the single-cell level, we developed two novel statistical tools.
First, SCPattern [29] is used to identify stage-specific genes over time; and second, Wave-Crest is used to reconstruct the differentiation trajectory from the pluripotent state through mesendoderm to DE. Based on this highresolution temporal reconstruction, we detected presumptive DE cells characterized with CXCR4 and SOX17 expression as early as $36 \mathrm{~h}$ post differentiation. Focusing on this time point, Wave-Crest identified candidate genes that could function as pioneer regulators governing the transition from mesendoderm to the DE state.

Owing to known technical variability and stochastic expression in single-cell gene expression measurements [30-33], rigorous functional validation of scRNA-seq analyses is essential. In order to specifically validate our analysis, we engineered a $T-2 A-E G F P$ reporter ES cell line by CRISPR/Cas9-mediated knock-in. Of all the candidate genes tested, we found that siRNA knockdown of KLF8 rendered one of the most overt delays in differentiation. A converse gain-of-function experiment demonstrated that KLF8 plays a previously unrecognized role during the transition from a $T^{+}$state to a $C X C R 4^{+} \mathrm{DE}$ state. Our results reveal that elevated levels of KLF8 enhance expression of DE markers but not mesodermal genes, suggesting that KLF8 acts specifically on the transition from mesendoderm to DE but not to mesoderm. Altogether, our study reinforces the importance of combining single-cell analysis and genetic approaches. We believe this strategy could be directly applied to other lineages during any differentiation paradigm to examine cell fate decisions.

\section{Results}

\section{scRNA-seq reveals a unique endoderm progenitor} signature

To begin investigating lineage-specific transcriptomic features at single-cell resolution, we performed a cohort of scRNA-seq experiments profiling snapshots of lineagespecific progenitor cells differentiated from $\mathrm{H} 1$ human ES cells using our established differentiation protocols, all adapted to chemically-defined culture conditions $[17,20,34,35]$. In order to obtain a high purity of lineage-specific progenitors, cells were enriched by fluorescence-activated cell sorting (FACS) with their respective markers (see details in "Methods" and Additional file 1: Figure S1). Progenitors differentiated from human ES cells included neuronal progenitor cells (NPCs, ectoderm derivatives, $\mathrm{n}=173$ ), DE cells (endoderm derivatives, $\mathrm{n}=138$ ), endothelial cells (ECs, mesoderm derivatives, $\mathrm{n}=105$ ), and trophoblast-like cells (TBs, extraembryonic derivatives, $n=69$ ). Single undifferentiated H1 $(n=212)$ and H9 $(n=162)$ human ES cells and human foreskin fibroblasts (HFFs, $\mathrm{n}=159$ ) were also included as controls. In total, 1018 single cells were analyzed in this cohort of experiments (Fig. 1a and Additional file 1: Figure S1). 

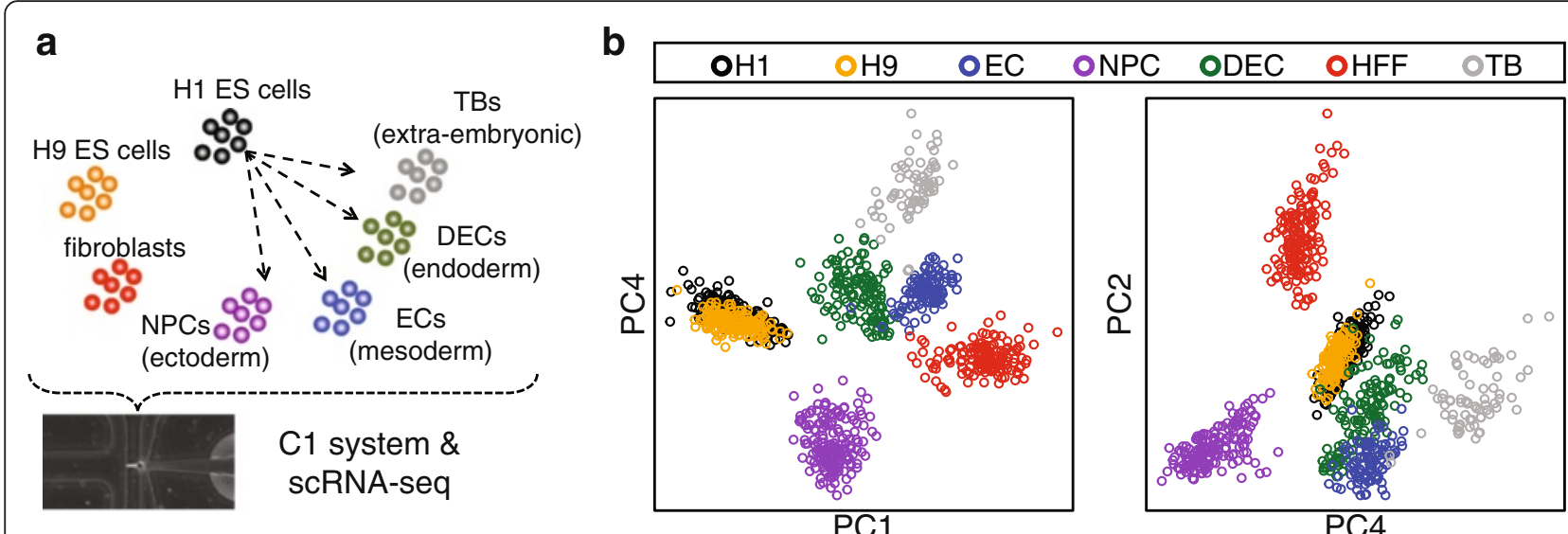

C

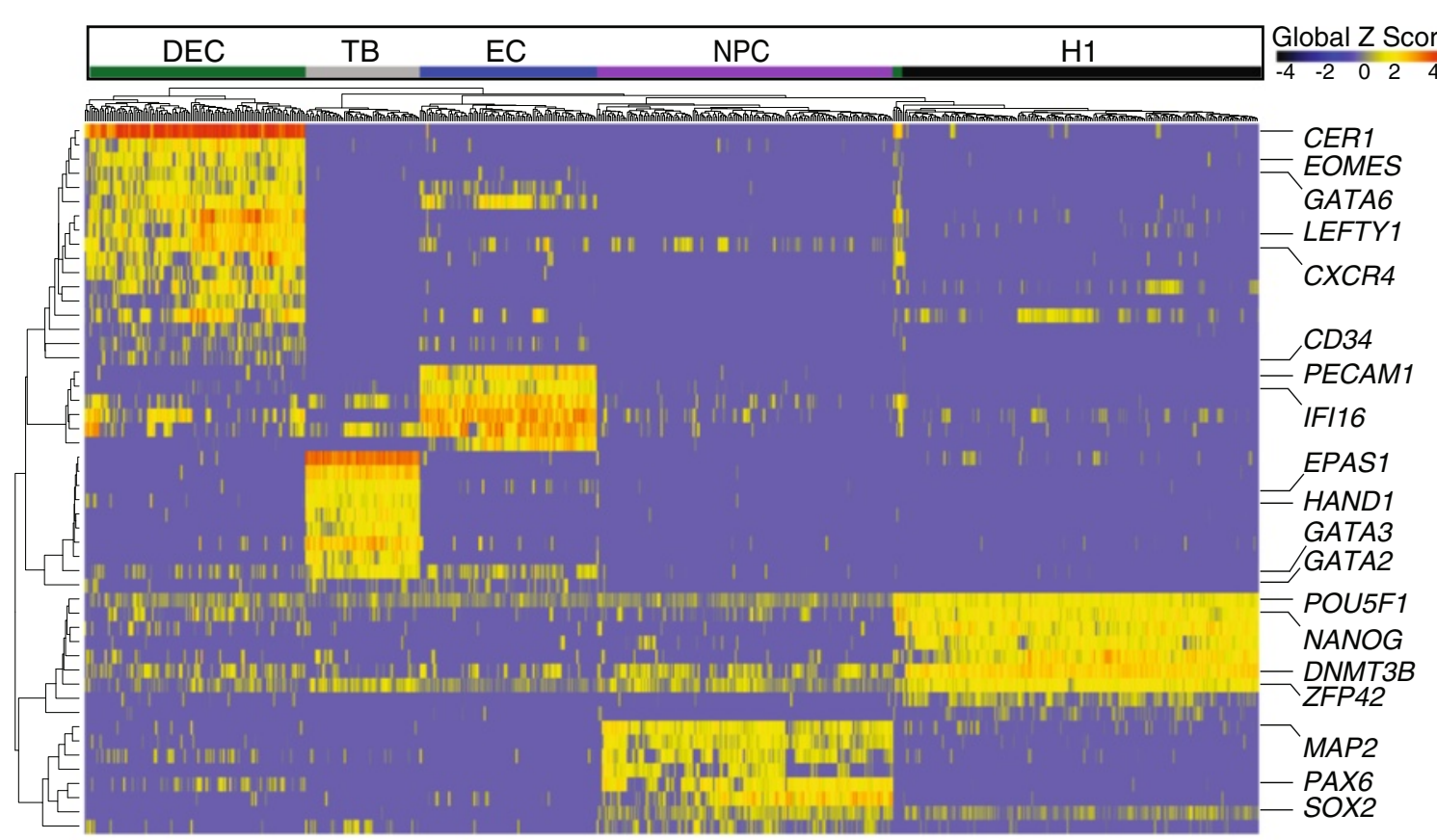

d

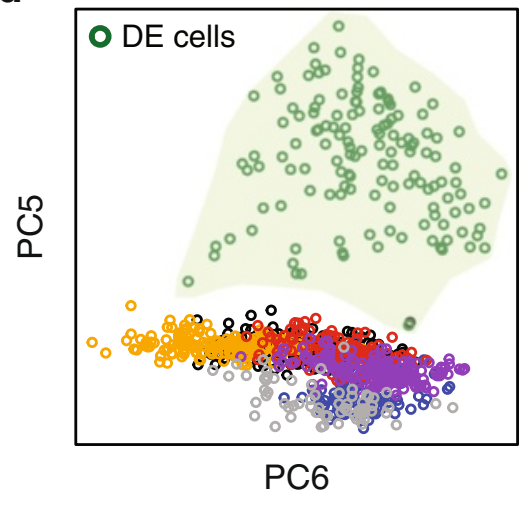

e

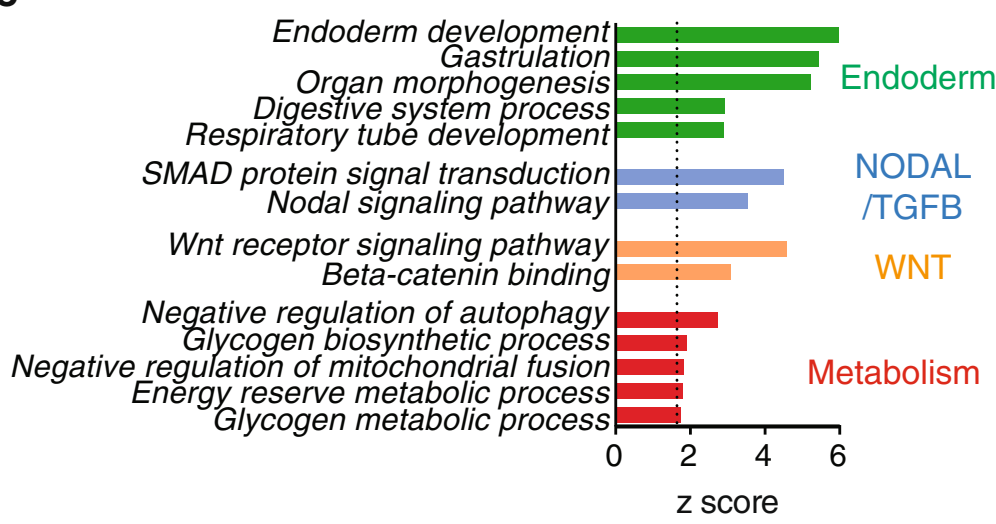

Fig. 1 (See legend on next page.) 
(See figure on previous page.)

Fig. 1 Snapshot scRNA-seq analysis of human ES-derived progenitors. a Schematics of experimental strategy. Human ES-derived lineage-specific progenitors were profiled at the single-cell resolution. b Principal component analysis (PCA) of all the cell types profiled. Shown are PC4 vs. PC1 and PC2 vs. PC4. c Hierarchical clustering analysis of progenitors differentiated from $\mathrm{H} 1$ cells with selected lineage-specific markers shown on the right. $\mathbf{d}$ PCA of all the cell types profiled, shown are PC5 vs. PC6. The light green shade highlights all the single DE cells. e Enrichment analysis of PC5 shown in (d). Bar graph shows the significant z scores of selected GO terms. Summary on collections of GO terms are shown on the right. Dashed line indicates statistical significant threshold at z score $=1.62$ (one tailed $p$ value $<0.05$ ). NPC neuronal progenitor cell, DEC definitive endoderm cell, EC endothelial cell, TB trophoblast-like cell, HFF human foreskin fibroblasts

To elucidate the distinctions between different lineages, we conducted bulk-projected principal component analysis (PCA), which projects individual cells on bulk RNA-seq defined principal component (PC) axes (see details in "Methods"). The majority of the single cells cluster according to their respective developmental lineages (Fig. 1b). The tight clusters of overlapped $\mathrm{H} 1$ and H9 single cells indicated the relative homogeneity of human ES cells compared to the rest of the progenitors. NPCs (ectoderm), TBs (extraembryonic), and HFFs (fibroblasts) were distantly positioned on the PCA plot while ECs and DE cells showed some overlapped domains, suggesting a closer lineage relationship. This result is consistent with the notion that mesoderm and DE cells may arise from a common progenitor pool during development and differentiation [20,36,37]. Hierarchical clustering analysis of key lineage markers further demonstrates distinct but rather uniform expression within each progenitor state (Fig. 1c). Specifically, all of the single undifferentiated $\mathrm{H} 1$ cells uniformly expressed high levels of pluripotency markers including POU5F1, NANOG, DNMT3B, and ZFP42 (REX1). By contrast, NPCs are enriched for the expression of SOX2, PAX6, and MAP2; ECs are enriched for PECAM1 and CD34; TBs are enriched for GATA3 and HAND1; and DE cells are enriched for CER1, EOMES, GATA6, LEFTY1, and CXCR4 (Additional file 2: Table S1). These analyses indicate that scRNA-seq of the progenitors is competent to reveal lineage-specific transcriptomic features.

The bulk-projected PCA shows that the majority of variation could be captured by the first five PCs (Additional file 1: Figure S2). Interestingly, PC5 clearly separates DE cells from all the other progenitors (Fig. 1d). This result suggests that PC5 gene loadings are a signature exclusively exhibited by differentiating $\mathrm{DE}$ cells. We also observed single DE cells distributed along the PC5 axis, indicating that this gene loading captured a heterogeneous or asynchronous pool of DE cells. Moreover, DE cells showed the greatest heterogeneity compared to the other three types of progenitors by correlation analysis (Additional file 1: Figure S2). To determine the signaling pathways associated with the $\mathrm{DE}$ signature, we performed Gene Ontology (GO) analysis utilizing the Allez algorithm, which used absolute gene loadings to weight gene-specific contribution to PC5 (see "Methods") [38]. Allez enrichment analysis identified endoderm development, organ morphogenesis, NODAL signaling pathway, regulation of WNT receptor signaling pathway, and energy reserve metabolic processes among the significantly enriched GO terms (Fig. 1e and Additional file 3: Table S2). While it is well established that both NODAL and WNT signaling are crucial for endoderm development [1-3, 11], little is known about how the metabolic state could influence DE differentiation. Based on these analyses, we investigated whether manipulating the metabolic conditions could impact DE differentiation.

\section{Acute hypoxic treatment enhanced DE differentiation}

It has been previously shown that lowering oxygen tension can reduce oxidative stress, shifting metabolic fueling pathways from oxidative phosphorylation to glycolysis to aid in maintaining pluripotency and reprogramming $[39,40]$. We therefore set out to measure the impact of lowering oxygen concentration in the cell culture microenvironment during the differentiation toward DE (see "Methods"). We chose to monitor the expression of Chemokine (C-X-C Motif) receptor 4 (CXCR4) in live cells as a surrogate marker for DE differentiation because: (1) CXCR4 expression is specific to DE but absent in the visceral endoderm compartment at the late primitive streak stage ( E7.5) mouse embryo [41]; and (2) human ESderived $\mathrm{CXCR}^{+}$cells have been shown to display hallmarks of DE [7, 15, 42].

At three days of differentiation, H1 or H9 ES cells cultured in severe hypoxia $\left(1.5 \% \mathrm{O}_{2}\right)$ significantly increased the percentage of CXCR4 ${ }^{+}$cells by FACS analysis compared to hypoxia $\left(5 \% \mathrm{O}_{2}\right)$ or normoxia $\left(20 \% \mathrm{O}_{2}\right)$ conditions (Fig. 2a and b). The percentages of cells coexpressing CXCR4 and SOX17 also increased in $1.5 \% \mathrm{O}_{2}$ condition (Additional file 1: Figure S3). Marker studies by quantitative real-time PCR (qPCR) analysis confirmed that the expression of pluripotency genes POU5F1, NANOG, and $S O X 2$ were effectively downregulated in all conditions (Fig. 2c). Importantly, key DE markers CXCR4, SOX17, $H N F 1 B, K I T$, and KRT19 were all significantly upregulated in $1.5 \% \mathrm{O}_{2}$ but not in $5 \% \mathrm{O}_{2}$, compared with normoxic conditions. Interestingly, hypoxic conditions also significantly suppressed $T$ expression, which is a 


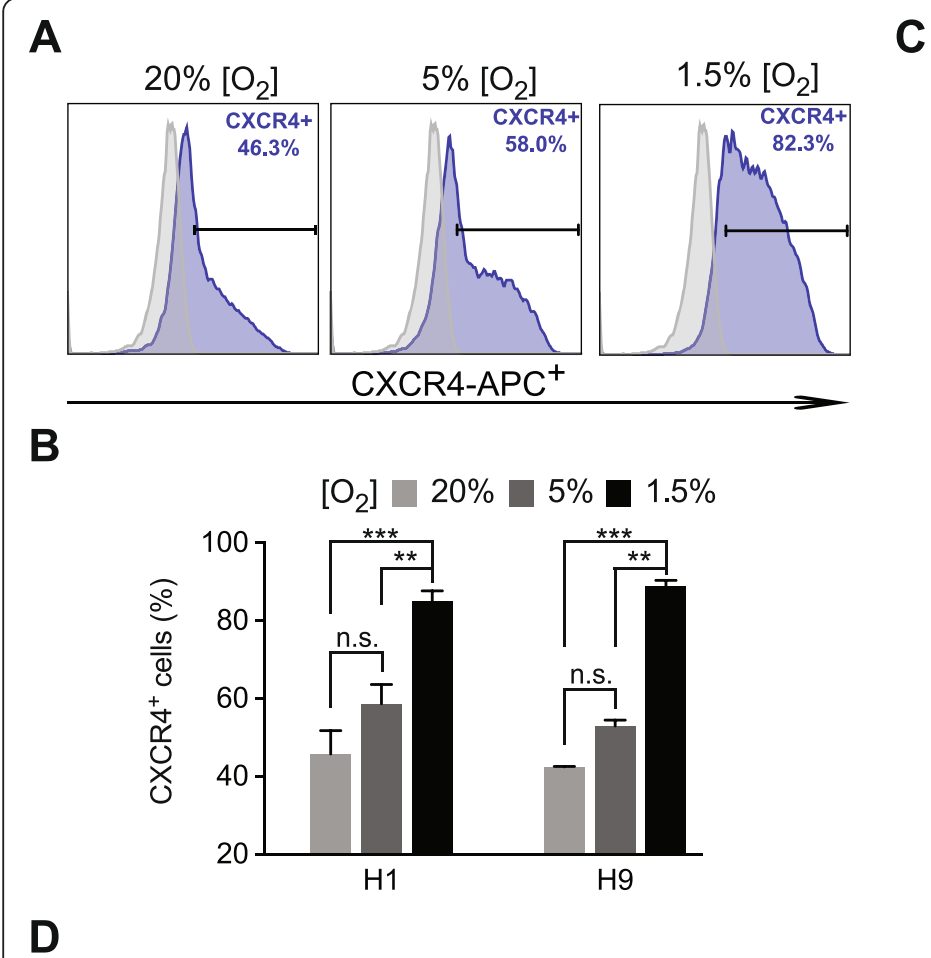

C
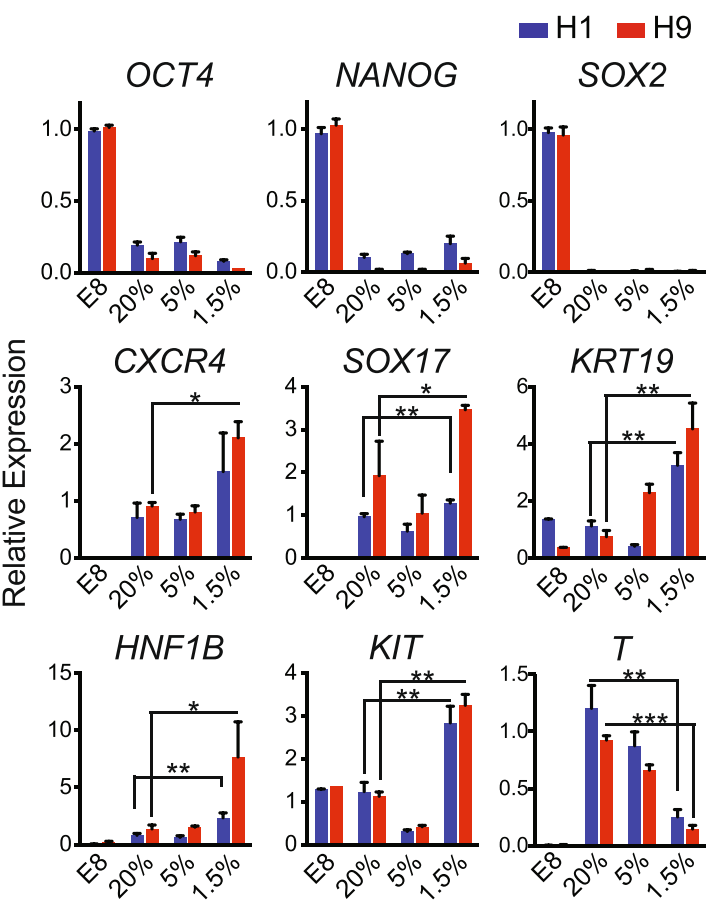

Days of differentiation
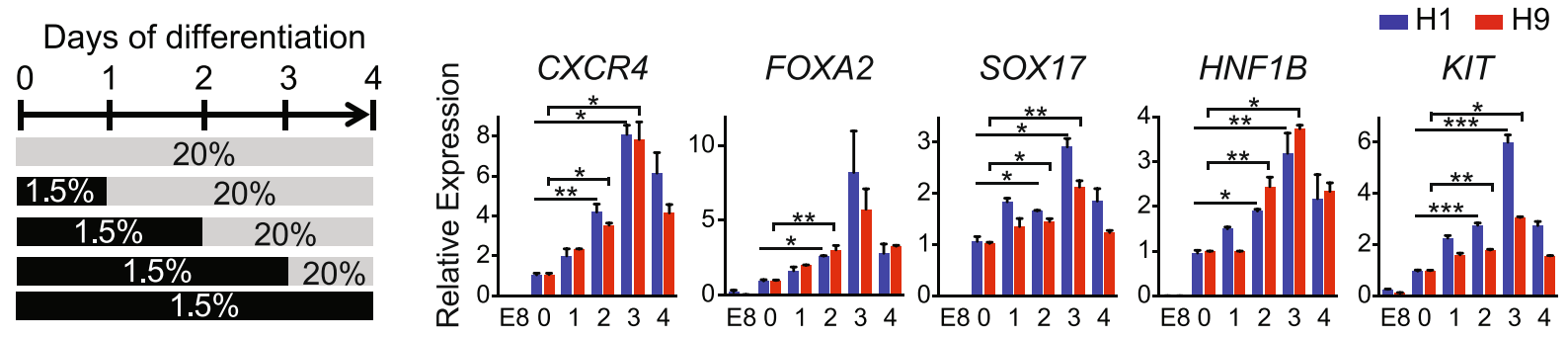

E

E8 undifferentiated

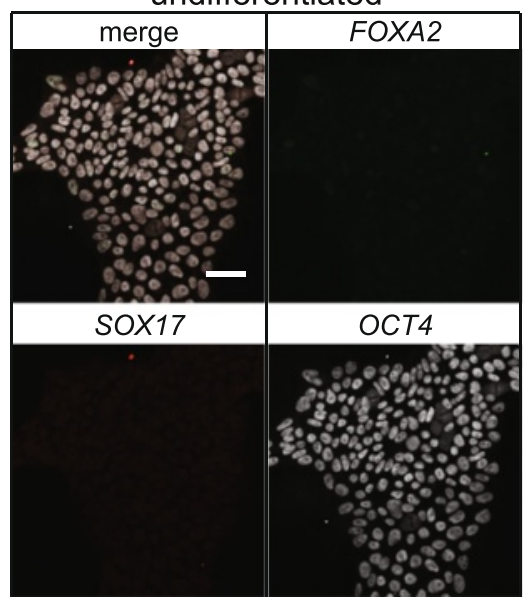

$20 \%\left[\mathrm{O}_{2}\right]$

$1.5 \%\left[\mathrm{O}_{2}\right]$
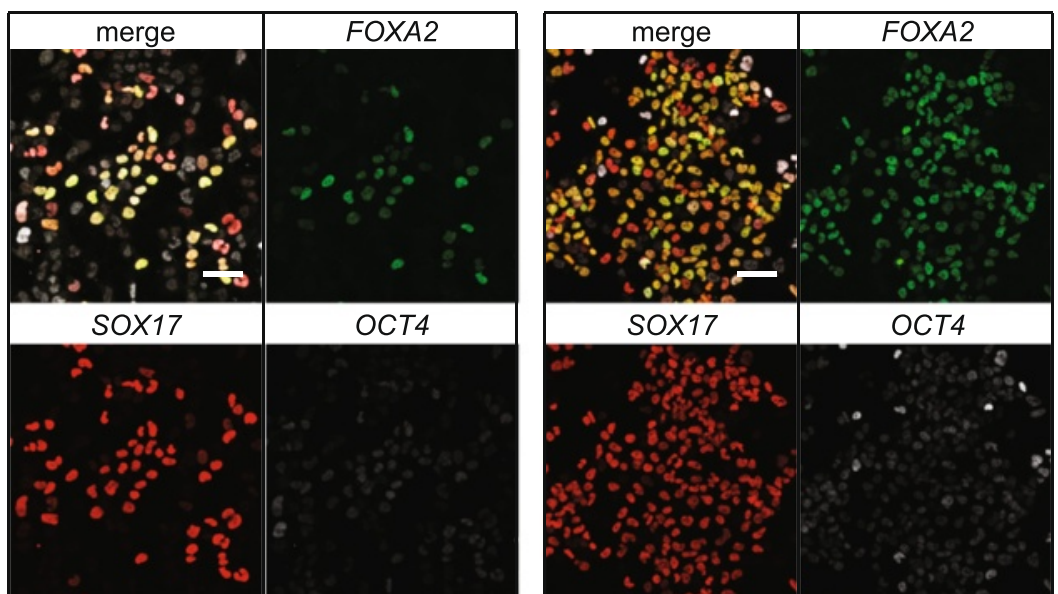

Fig. 2 (See legend on next page.) 
(See figure on previous page.)

Fig. 2 Acute hypoxic treatment enhanced DE formation. a FACS analysis of anti-CXCR4 staining of H1 cells differentiated for three days towards (blue population) under various hypoxia conditions. Undifferentiated $\mathrm{H} 1$ cells were gated as negative controls (gray populations). The $x$-axis indicates the APC channel. b Summary of the percentages of $\mathrm{CXCR} 4^{+}$cells (with various oxygen concentrations) from $\mathrm{H} 1$ or $\mathrm{H} 9$ differentiation for three days toward DE cells. c QPCR analysis of experiments performed in (b). All expression levels were first normalized to endogenous GAPDH. For pluripotency markers (upper panels), samples were normalized to undifferentiated $\mathrm{H} 1$ or $\mathrm{H} 9$ samples, which were arbitrarily set to 1 . For other markers (mid and lower panels), samples were normalized to the $20 \% \mathrm{O}_{2}$ samples, which were arbitrarily set to 1. d Left panel, schematics of various lengths of hypoxic treatment. Right panel, GPCR analysis at day four of differentiation for $\mathrm{H} 1$ or $\mathrm{H} 9$ cells. The $x$-axis indicates the number of days treated with $1.5 \% \mathrm{O}_{2}$ as indicated in the left panel. Samples were normalized to those from normoxia, which were arbitrarily set to 1. e Confocal images of OCT4, FOXA2, and SOX17 immunofluorescence staining at day two of differentiation under $20 \%$ or $1.5 \% \mathrm{O}_{2}$. Scale bars $=50 \mu \mathrm{m}$. All data are mean \pm S.D. ${ }^{* * *} p<0.001 ;{ }^{* *} p<0.01 ;{ }^{*} p<0.05$, by one-tailed t-test. $n$. s. no significant difference

pan-mesendoderm marker whose expression precedes DE marker expression (Fig. 2c).

While differentiating human ES cells with $1.5 \% \mathrm{O}_{2}$ has a profound effect on the induction of DE markers, we also observed that proliferation decreased and cell death increased after prolonged hypoxic differentiation (Additional file 1: Figure S3). Thus, we speculate that $1.5 \% \mathrm{O}_{2}$ treatment stimulates the emergence of nascent DE cells but may not be beneficial for long-term proliferation and maturation. To determine the critical timing when the effects of severe hypoxia could take place, we performed acute-hypoxia treatment in our differentiation protocols (Fig. 2d). In this set of experiments, H1 or $\mathrm{H} 9 \mathrm{ES}$ cells were first differentiated in $1.5 \% \mathrm{O}_{2}$ environment for various lengths (zero, one, two, three, or four days of differentiation) and then switched back to normoxic conditions for up to four days of differentiation (Fig. 2d). Consistently, qPCR analysis showed that continuous hypoxic treatment throughout the first three days of differentiation resulted in substantially enhanced expression of key DE markers, such as CXCR4, FOXA2, SOX17, HNF1B, and KIT (Fig. 2d). Remarkably, the marker expression showed significant upregulation within just two days of hypoxic treatment. Immunofluorescence staining of SOX17 and FOXA2 confirmed areas with an increased number of $\mathrm{SOX}_{17}{ }^{+}$and FOXA2 ${ }^{+}$ cells within two days of severe hypoxic differentiation condition compared to normoxia control (Fig. 2e). These results corroborate a recent mouse ES cell study in which hypoxia culture facilitated DE differentiation [43]. Most importantly, this observation suggests that the birth of nascent DE cells is a well-timed event. Intervention of this process by enhancing factors (in this case, with severe hypoxia) allows us to close in on the key moments in which DE cells become specified from their mesendoderm precursors. These results motivated us to closely examine the transition from mesendoderm to DE state at a higher temporal resolution.

\section{Reconstruction of temporal single-cell states identifies regulators for nascent $D E$ cell differentiation}

To precisely pinpoint the staging and timing during DE emergence, we performed scRNA-seq at time points along the differentiation protocol to produce DE cells from human ES cells (see "Methods"). A total of 758 single cells were captured and profiled by scRNA-seq at 0 , 12, 24, 36, 72, and $96 \mathrm{~h}$ of differentiation (Fig. 3a). PCA revealed that single cells from each time point along the differentiation course occupied a unique dimensional space, indicating a robust directional differentiation (Fig. 3a and Additional file 1: Figure S4). However, we noticed overlapping domains between single cells collected from 72 and $96 \mathrm{~h}$ of differentiation, indicating a similar transcriptome profile (see "Discussion").

To further characterize the genes participating in each stage of differentiation, we performed differential expression analysis by SCPattern, which identifies significantly upregulated or downregulated genes between each pair of adjacent sampling time points (see "Methods"). SCPattern is a novel algorithm developed for differential expression analysis on time course scRNA-seq data [29]. Existing differential expression tools developed for bulk RNA-seq make distributional assumptions that are inappropriate for scRNA-seq data. In general, for a given gene, the bulk RNA-seq methods usually assume expression values within a biological condition follow a unimodal distribution such as negative binomial distribution [44-48] or Poisson distribution [49]. However, such assumptions are often violated in scRNA-seq data due to the present of sub-populations and technical dropouts $[50,51]$. SCPattern makes no parametrical assumptions on the distribution of the single-cell gene expression values; instead, it performs non-parametric tests based on a KolmogorovSmirnov statistic and is able to detect various types of changes over multiple ordered conditions [29].

At $12 \mathrm{~h}$ of differentiation, the majority of the cells responded to BMP4, Activin A, and CHIR 99021 (small molecule used as a WNT signaling agonist) treatment by robustly expressing NODAL, EOMES, and ID1. At $24 \mathrm{~h}$ of differentiation, a second wave of genes exhibited high levels of expression such as T, MSX2, and CDX1, all indicating a transition of the cells towards a primitive streak state. At $36 \mathrm{~h}$ of differentiation, the level of $T$ transcripts rapidly decreased, characterized by upregulation of early DE-specific genes, such as CER1 and GATA4. At $72 \mathrm{~h}$ of differentiation, the majority of the 


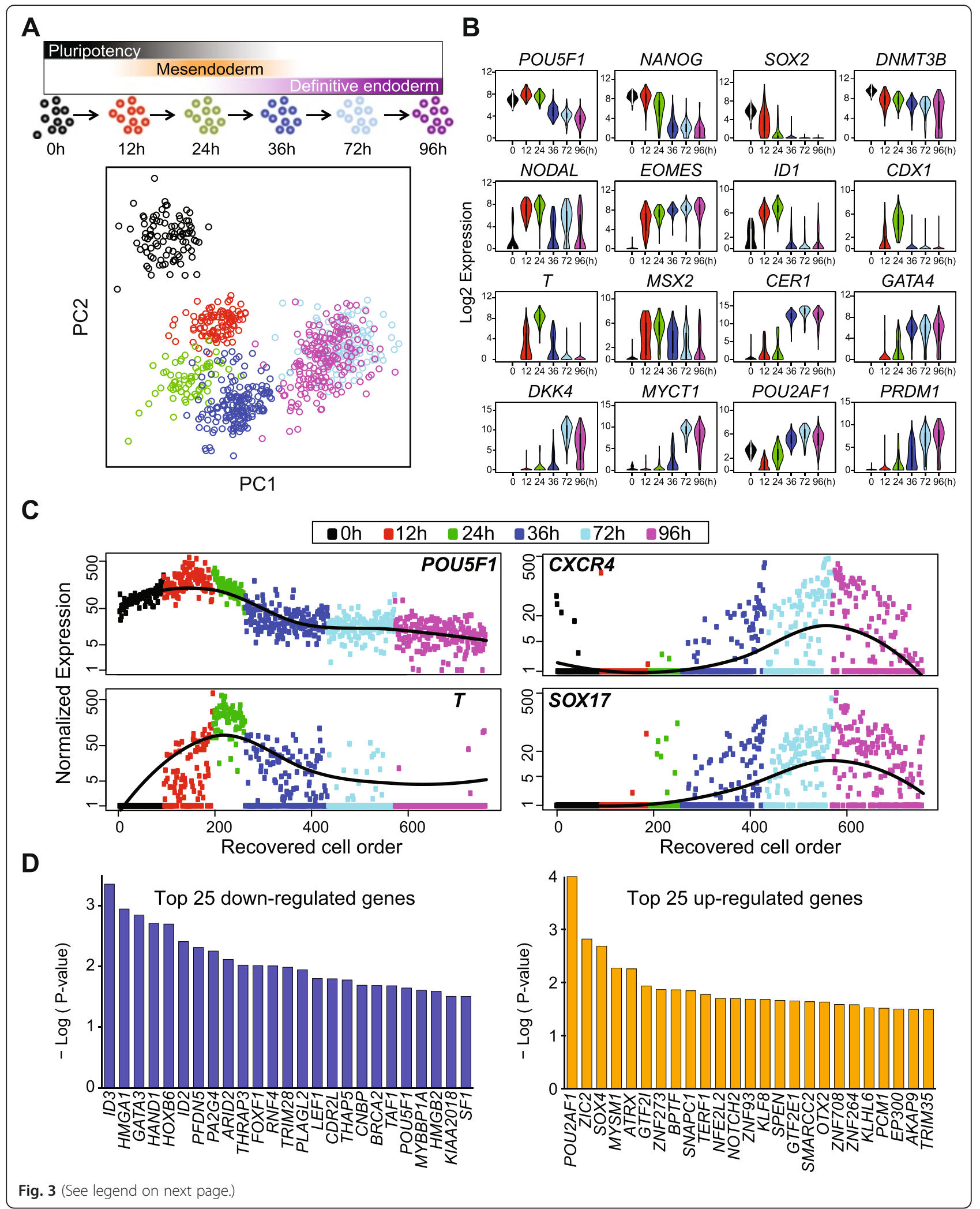


(See figure on previous page.)

Fig. 3 Time course scRNA-seq data analysis and reconstructing DE differentiation trajectory. a Upper panel, schematics of experimental strategy illustrating time points of scRNA-seq sampling along the differentiation from pluripotent state though mesendoderm to DE cells. Lower panel, PCA of scRNA-seq data, shown is PC1 vs. PC2. $\mathbf{b}$ Violin plots of selected stage-specific markers identified by SCPattern analysis. The $y$-axis indicates normalized expression value, Log2(expected count +1 ). The $x$-axis indicates time points of sampling. $\mathbf{c}$ Reconstructed single-cell order by Wave-Crest. Shown are four of the eight genes used for temporal reconstruction. The $x$-axis indicates cells following Wave-Crest recovered cell order. The $y$-axis indicates normalized expression value. Fitted lines of gene-specific expression are shown in black. $\mathbf{d}$ Top 25 upregulated or downregulated genes identified by Wave-Crest at $36 \mathrm{~h}$ of differentiation. The $x$-axis indicates gene symbol, the $y$-axis indicates the significance value, -Log ( $p$ value) of each gene

cells expressed endogenous DKK4 and MYCT1. Key DE markers continue to be expressed at high levels among single cells at the 96-h time point, including EOMES, CER1, GATA4, PRDM1, and POU2AF1, indicating that cells progressed toward the DE state [52, 53] (Fig. 3b and Additional file 4: Table S3).

At any time point during a differentiation protocol, each sampled cell is not necessarily identical to the others; likely a result of differences in the cell cycle and the local microenvironment of the differentiating cells. We capitalized on the asynchronous nature of the cells to reconstruct a single-cell order following the differentiation trajectory toward DE. By reconstructing this single-cell transcriptomic order, we hoped to identify novel regulators whose expression could mediate the transition from mesendoderm toward a DE state. We devised a novel statistical tool, Wave-Crest, to reorder single cells according to the expression of key gene markers. The cell order reconstruction step of Wave-Crest takes a group of genes of interest and aims to recover a smooth expression profile along time for each of the genes in consideration. To do so, Wave-Crest implements a constrained extended nearest-insertion (ENI) algorithm to reorder cells within each time point utilizing boundary information from other time points. In particular, if a cell's expression profile is closer to the cells from the previous (next) time point, the cell will be placed in an earlier (later) position in the reconstructed order. The reordering is under the constraint that cells from different collection times are not allowed to be mixed in the recovered order. After ENI, Wave-Crest utilizes the 2-opt algorithm to further refine the cell order (see "Methods") [54]. When applied WaveCrest to the scRNA-seq DE differentiation time course data, we selected the genes of interest by combining empirical results from SCPattern and prior knowledge $[1,2,5,7,9,20]$. Our reconstruction focus on markers representing the pluripotent, mesendodermal, and DE states to build a directional reordering of single cells without characterizing the branching structure of single cells (Additional file 1: Figure S5). In particular, pluripotency marker POU5F1 gradually decreased over the course of $96 \mathrm{~h}$ of differentiation, whereas mesendoderm marker $T$ expression first peaked at $24 \mathrm{~h}$ and gradually decreased at 36-72 $\mathrm{h}$ of differentiation (Fig. 3c, left panel). Remarkably, in the reconstructed order, CXCR4 and SOX17 both showed a subtle but significant upregulation as early as $36 \mathrm{~h}$ of differentiation and continued to increase in later time points (Fig. 3c, right panel). Importantly, in the recovered cell order at the 36 -h time point, $C X C R 4^{+}$and $S O X 17^{+}$single cells appear later and are almost mutually exclusive to the POU5F $1^{\text {high }}$ and $T^{\text {high }}$ cells, indicating our reconstructed cell order is indeed aligned with the differentiation trajectory toward a DE fate (Additional file 1: Figure S5). These results also suggest that the presumptive DE transcriptional program begins between 24 and $36 \mathrm{~h}$, which is surprisingly early considering most established human pluripotent stem cell protocols typically consider DE cells to emerge around days 4 or 5 of differentiation [7, 11, 15, 42, 52].

The second step of Wave-Crest involves application of polynomial regression models to identify genes whose expression profile best fits this reconstructed differentiation trajectory, a strategy we call "fishing" (see "Methods"). We focused on fishing genes at $36 \mathrm{~h}$ of differentiation because this appears to be the transition time characterized with a steep downregulation of POU5F1 and $T$ as well as upregulation of CXCR4 and SOX17 (Fig. 3c and Additional file 1: Figure S5). Wave-Crest extracted the reconstructed cell order from the 172 cells collected at $36 \mathrm{~h}$ of differentiation and then fished against a curated list of transcriptional regulators (Additional file 5: Table S4). The top-fished genes were defined as the genes which had small fitting error in the polynomial regressions. Permutation tests were applied to infer the significance. These top-fished genes were then classified into upregulated and downregulated groups by the coefficient sign of gene-specific slope fitting. The top-fished gene list included known markers for mesendoderm or mesoderm (downregulated genes), such as GATA3 (No. 3), HAND1 (No. 4), FOXF1 (No. 11), LEF1 (No. 15), and markers for DE specification (upregulated genes), such as SOX4 (No. 3) and OTX2 (No. 18), further demonstrating the power of Wave-Crest reconstruction (Fig. 3d and Additional file 6: Table S5). We reasoned that the top upregulated genes are likely to promote the transition from mesendoderm to DE (Fig. 3d). To test these genes, a 
reporter was devised to measure the effect of manipulation of candidate genes during the transition from a $T^{+}$ state to a $C X C R 4^{+}$state in live cells.

H9-T-2A-EGFP reporter line is a robust tool to monitor the mesendoderm to $D E$ transition

In order to measure the levels of real-time endogenous $T$ protein expression, we inserted a $2 A$-EGFP-PGK-Puro cassette into the endogenous $T$ locus via CRISPR/Cas9mediated gene targeting (Fig. 4a) [55, 56]. Collective analyses from copy number qPCR, junction PCR, and southern blotting confirmed that clone 39 was a correctly targeted clone with only one copy of the EGFP and Puro cassette knock-in into the endogenous $T$ locus. (Fig. 4b and Additional file 1: Figure S6). Upon removal of the PGK-Puro cassette with transient Cre expression, this T-2A-EGFP line (clone 39) was used in all the subsequent experiments. Cytogenetic test also verified a normal karyotype after gene targeting and clonal expansion (Additional file 1: Figure S6). Importantly, qPCR analysis

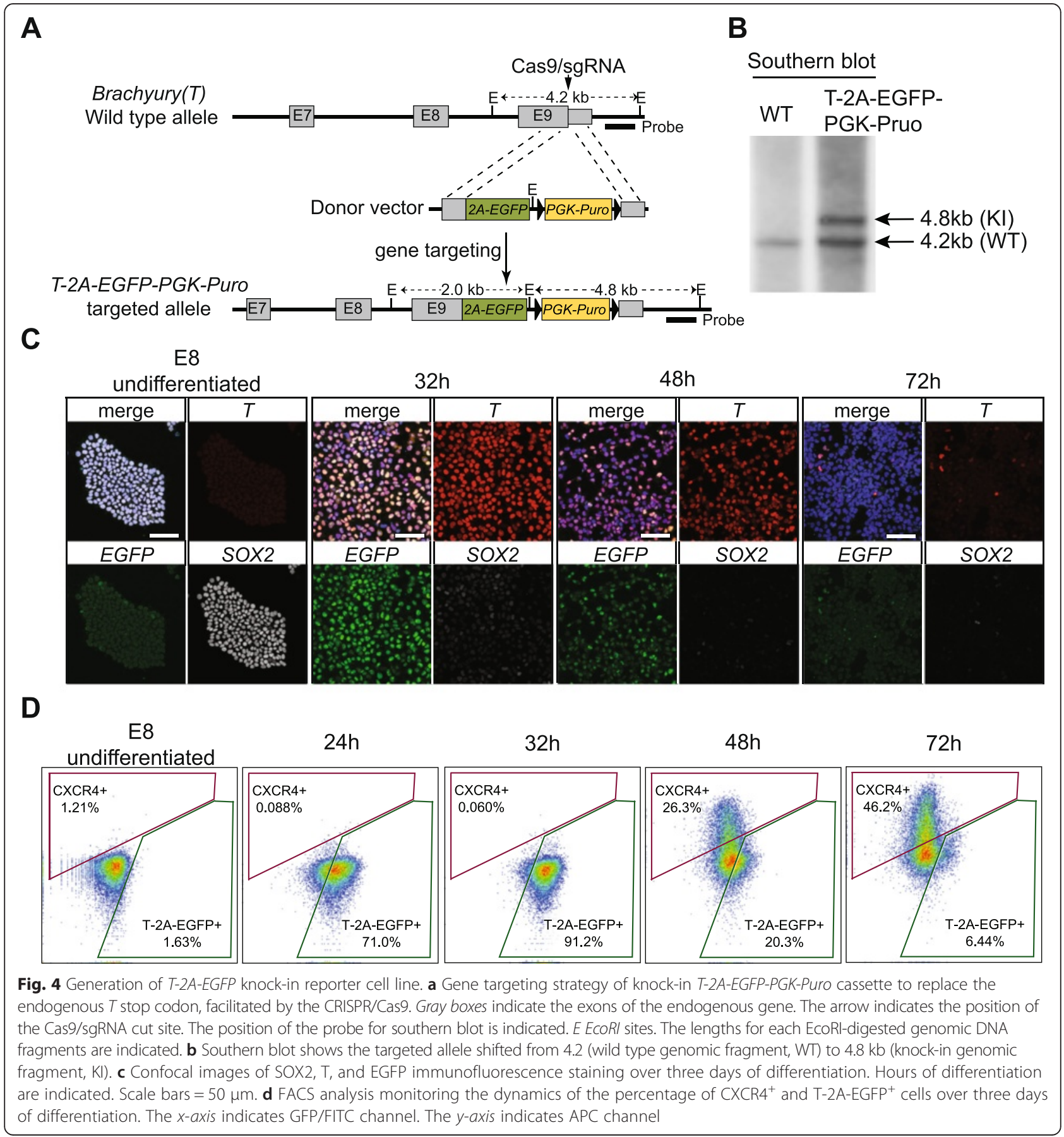


on EGFP-sorted cells and immunofluorescence staining confirmed that the dynamics of EGFP expression is highly correlated with both the endogenous $T$ transcript and protein expression, respectively, making the $T-2 A$ EGFP reporter a robust readout for $T^{+}$state in the course of our DE differentiation (Fig. 4c and Additional file 1: Figure S6).

Simultaneously measuring both $T$ and $C X C R 4$ protein levels by FACS provided both the precision and resolution required to detect cell state transitions. After just $24 \mathrm{~h}$ of differentiation, more than $70 \%$ of the cells became T-2A-EGFP ${ }^{+}$. The percentage of T-2A-EGFP ${ }^{+}$cells reached a zenith (approximately $90 \%$ ) between 28 and $32 \mathrm{~h}$ of differentiation and then gradually decreased over time ( $20 \%$ at $48 \mathrm{~h}$ and below $10 \%$ at $72 \mathrm{~h}$ of differentiation, Fig. 4d). Co-staining cells with anti-CXCR4 antibodies revealed that less than $1 \%$ of the cells were $\mathrm{CXCR}_{4}^{+}$at $24 \mathrm{~h}$ of differentiation. After $48 \mathrm{~h}$, approximately $25 \%$ of the cells became CXCR $4^{+}$, increasing to above $40 \%$ after $72 \mathrm{~h}$ of differentiation (Fig. $4 \mathrm{~d}$ ). Therefore, while $\mathrm{T}$ expression transiently peaked around $32 \mathrm{~h}$, CXCR4 expression continually increased, starting as early as $36-40 \mathrm{~h}$ of differentiation, a dynamic expression pattern mirroring the temporal profiling by scRNA-seq described above (Fig. 3c). Thus, monitoring the expression of T-2A-EGFP and CXCR4 over time presented a tractable means to screen candidate genes identified in the Wave-Crest analysis.

\section{KLF8 mediates the mesendoderm to DE transition}

We hypothesized that if a regulator plays a promotional role during the transition from mesendoderm toward $\mathrm{DE}$, then knocking down its expression would delay this progression. On the other hand, overexpression of such regulators should accelerate this progression. We conducted a siRNA knockdown screen focused on the top 25 upregulated genes identified by Wave-Crest (Fig. 3d). We accounted for both the percentages of $\mathrm{EGFP}^{+}$and $\mathrm{CXCR}_{4}^{+}$of each gene knockdown at day two of differentiation (between 45 and $48 \mathrm{~h}$ ), defined as a "Differentiation Score" for each gene tested (see "Methods"). When compared with non-target siRNA controls (Differentiation Score arbitrarily set to 1), knockdown of CXCR4 reduced the percentage of $\mathrm{CXCR} 4^{+}$cells from greater than $30 \%$ to approximately $10 \%$; whereas knockdown of $T$ substantially reduced the percentage of $\mathrm{EGFP}^{+}$cells and increased percentage of $\mathrm{CXCR} 4^{+}$cells, validating the efficacy of siRNA knockdown protocols (Fig. 5a). Among all the genes tested, TERF1, SPEN, and KLF8 knockdowns displayed the lowest Differentiation Score (i.e. the most potent blockade of differentiation) (Fig. 5b). Between the top three genes, KLF8 knockdown showed the smallest increase of T-2A-EGFP expression, indicating a more specific blockade of DE differentiation rather than enhancing mesendoderm or mesoderm differentiation (Fig. 5a and Additional file 1: Figure S7). KLF8 is expressed at a much lower level in undifferentiated human ES cells than TERF1 or SPEN (Additional file 1: Figure S7). KLF8 was also detected as a differentially expressed gene in our SCPattern analysis (Additional file 4: Table S3). Collectively, these data support the idea that $K L F 8$ may play a specific role during the transition from mesendoderm toward DE cells.

To further examine this possibility, we generated a doxycycline (DOX)-inducible $K L F 8$ transgene integrated by the PiggyBac system [57] into the T-2A-EGFP reporter cell line. Upon differentiation, induction of KLF8 (DOX treatment from 20 to $40 \mathrm{~h}$ of differentiation) robustly increased the percentage of CXCR4 ${ }^{+}$cells from $\sim 5 \%$ to above $40 \%$ in a dosage-dependent manner (Fig. 5c), accompanied by the loss of T-2A-EGFP ${ }^{+}$cells (Fig. $5 \mathrm{c}$ ). This result was confirmed by examining a total of three independent KLF8 overexpression clones. Furthermore, DOX induction of $K L F 8$ led to significant activation of $\mathrm{DE}$ markers including CXCR4, HNF1B, SOX17, and KIT, as judged by qPCR analysis from all three clones (Fig. $5 \mathrm{~d}$ ). Importantly, KLF8 overexpression did not induce the expression of mesendoderm or mesoderm markers such as T, EOMES, GSC, MSX2, and PDGFRA (Fig. 5d). We also observed that overexpressing KLF8 increased cell mobility as evident by the upregulation of TWIST1, an epithelialto-mesenchymal transition (EMT) marker (Fig. 5d). These results indicate that $K L F 8$ plays a specific role promoting the transition from $T^{+}$mesendoderm to $C X C R 4^{+} \mathrm{DE}$ fate, perhaps through the suppression of $T$ or by enhancing the EMT during DE differentiation. These results also suggest that the cell state transition from mesendoderm to DE is a complex and dynamic process, coupling the expression of specific transcriptional regulators with changes in cell migratory behavior. Altogether, our single-cell analysis identified $K L F 8$ as a previously unrecognized positive regulator of the transition from mesendoderm to nascent DE (Fig. 6). Other genes identified by the single-cell analysis are additional candidates for being regulators of this transition as well. We anticipate that the strategy used in this study - using scRNA-seq analysis to form hypotheses that can be tested by more conventional techniques may be further applied to uncover novel regulators in other lineages during cell fate decisions both in vitro and in vivo.

\section{Discussion}

\section{scRNA-seq from lineage progenitors and the role of hypoxia on DE differentiation}

Our scRNA-seq analysis of distinct progenitors derived from human ES cells revealed that one of the principal components (PC5) discriminated the DE cells from all other progenitors (Fig. 1d). Allez enrichment analysis on 


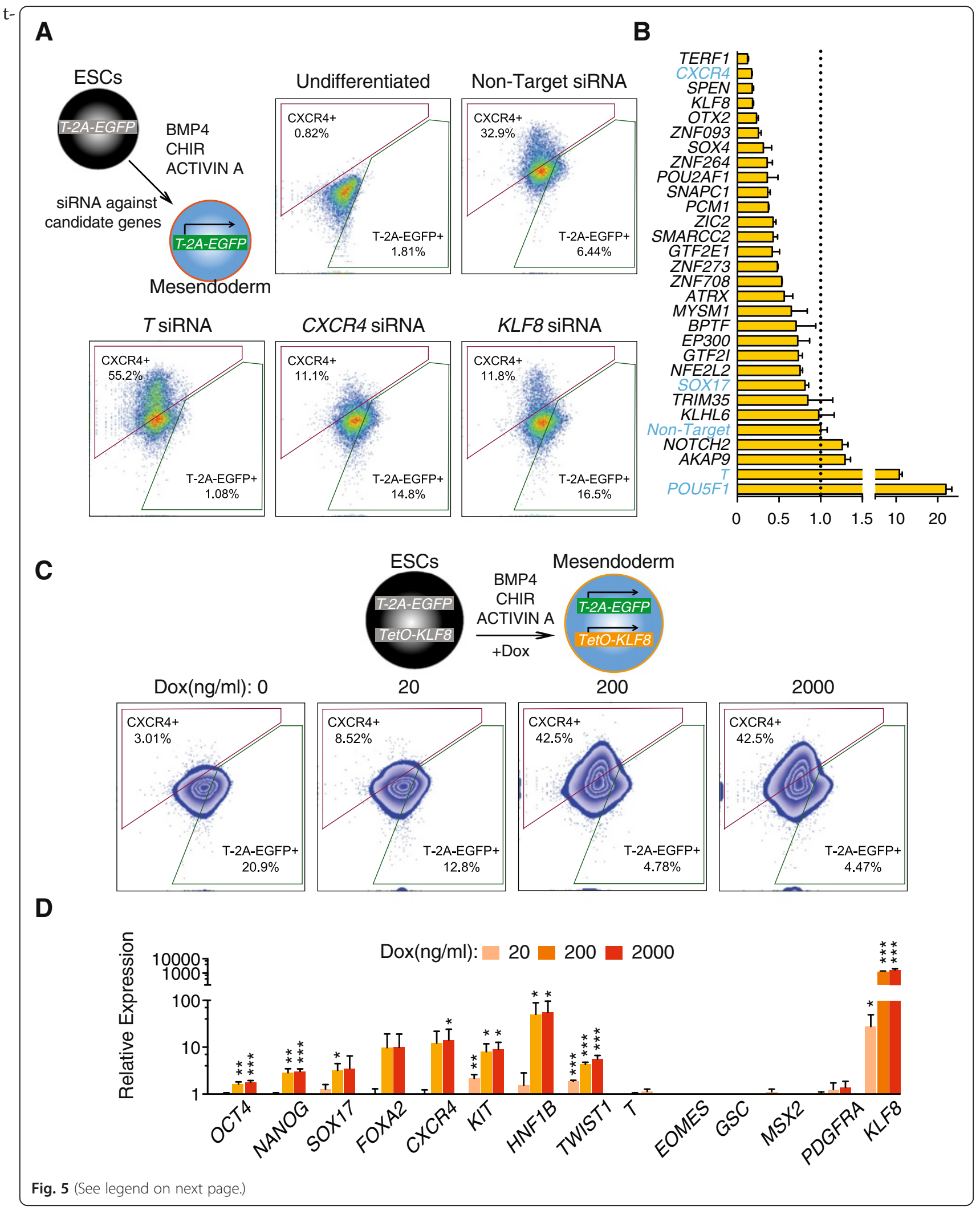


(See figure on previous page.)

Fig. 5 KLF8 mediates mesendoderm to DE differentiation. a Schematics of siRNA knockdown strategy. FACS analysis of CXCR4 and T-2A-EGFP expression at day two of differentiation with representative gene-specific siRNA transfection results. $\mathbf{b}$ Summary Differentiation Scores of siRNA knockdown experiments of the top 25 genes tested. The dashed line indicates a Differentiation Score of 1, set by the non-target siRNA control. Genes in blue font indicate control experiments. c Upper panel, schematics of KLF8 overexpression strategy during differentiation. Lower panel, FACS analysis shows CXCR4 and T-2A-EGFP expression at day two of differentiation. DOX Doxycycline. $\mathbf{d}$ QPCR analysis of three independent KLF8 overexpression clones tested at day two of differentiation. All data are shown as mean \pm S.D. ${ }^{* *} p<0.001 ;{ }^{* *} p<0.01$; ${ }^{*} p<0.05$, all by one-tailed ttest. In all FACS plots, the $x$-axis indicates GFP/FITC channel, the $y$-axis indicates APC channel

he gene loadings from PC5 revealed a role for metabolism in the genesis of DE cells (Figs. 1e and 2). Among the human ES cells tested, culture conditions of $1.5 \%$ $\mathrm{O}_{2}$, but not $5 \% \mathrm{O}_{2}$, consistently increased the percentage of CXCR4 ${ }^{+}$cells (Fig. 2b). Most importantly, the impact of hypoxia is pronounced in the first two days of differentiation (Fig. $2 \mathrm{~d}$ and e). Interestingly, only one recent report thus far described a positive effect of hypoxia on differentiating mouse ES cells to DE [43]. However, given that mouse and human ES cells are maintained by distinct signaling pathways $[58,59]$, it was not entirely clear if the hypoxiaenhanced DE differentiation may or may not directly translate to human ES system. To the best of our knowledge, our study is the first to investigate the effects of hypoxia during early definitive endoderm differentiation from human ES cells.
Most importantly, the implications from these results are twofold. First, there is a critical time window when the precursors of $\mathrm{DE}$ are responsive to hypoxic conditions, causing the initial upregulation of $\mathrm{DE}$ markers (Fig. 6). Second, it is plausible that cells destined to become DE cells, experience a lower oxygen concentration that prompts their specification to DE fate. Future whole embryo culture experiments will determine if a hypoxic microenvironment can enhance DE formation/specification.

\section{Temporal scRNA-seq analysis highlights the transcriptomic} transitions from pluripotent to mesendoderm to DE states We compared scRNA-seq profiles between each pair of neighboring time points over four days of differentiation (Fig. 3a) and found that the number of differentially expressed genes generally decreased over time (2224

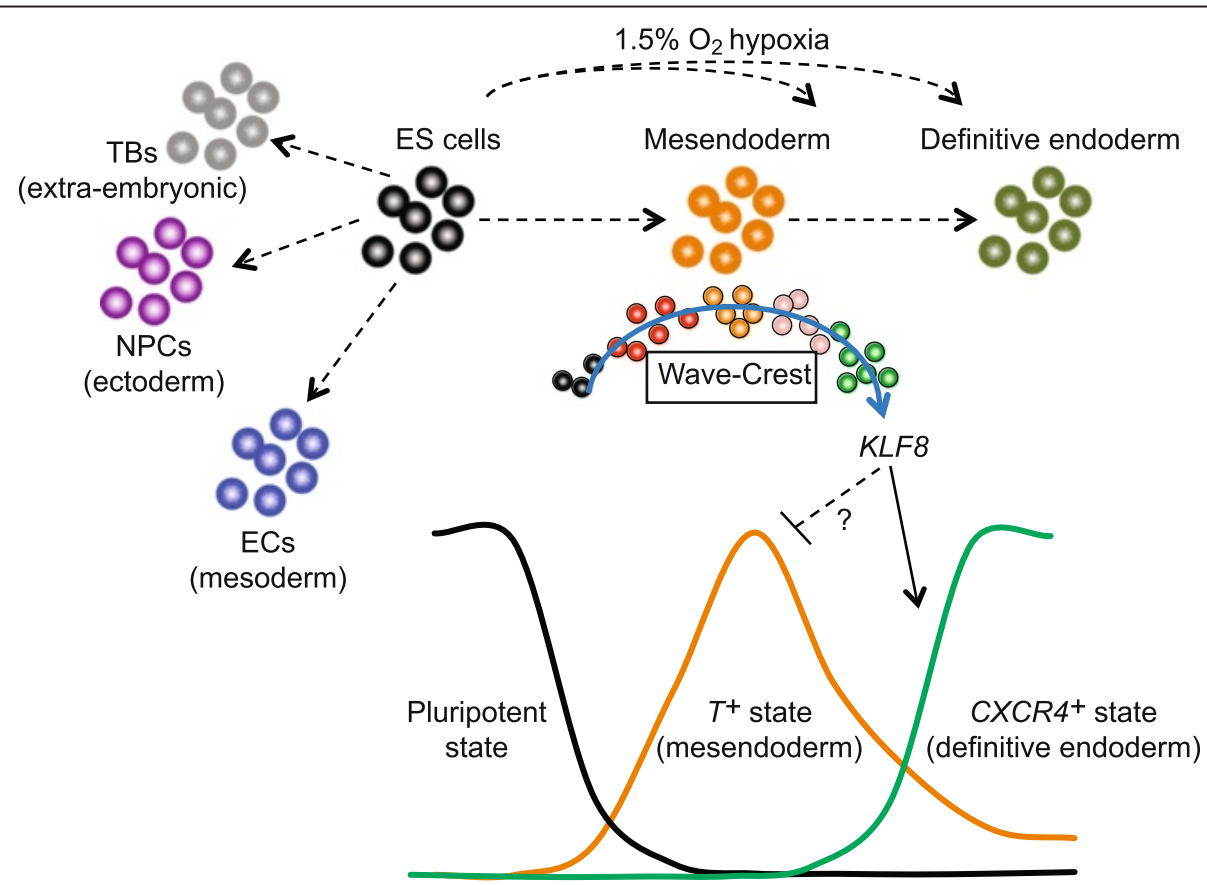

Fig. 6 Snapshot and temporal scRNA-seq profiling on progenitor cell states. scRNA-seq from snapshots of lineage-specific progenitors revealed that hypoxia enhances DE differentiation in a time-sensitive manner, likely acting on the mesendodermal progenitors. The differentiation trajectory was reconstructed by Wave-Crest (blue arrow) using time course scRNA-seq along the differentiation towards DE cells. Results from loss-of-function and gain-of-function experiments demonstrated that KLF8 function as a positive regulator mediating cell states transitions from $T^{+}$mesendoderm to $C X C R 4^{+}$DE cells. We hypothesize that KLF8 can suppress $T^{+}$state and in turn enhance the activation of CXCR4 $4^{+}$state. The molecular mechanisms of how KLF8 directly or indirectly suppress $T^{+}$state require future investigation 
genes $[0-12 \mathrm{~h}], 830$ genes $[12-24 \mathrm{~h}], 1317$ genes [24-36 h], 466 genes [36-72 h], and 11 genes (72-96 h)]. This observation suggests that transcriptional changes gradually decrease with continual BMP4 and Activin A signaling. Interestingly, our PCA plot (Fig. 3a) shows overlapped individual cells collected between $72 \mathrm{~h}$ and $96 \mathrm{~h}$, suggesting that cells could gradually transition into a relatively "stable" state at $72 \mathrm{~h}$ of differentiation.

When focusing on the $36 \mathrm{~h}$ time point of differentiation, the reconstructed cell order provides a glimpse of how the cells progress over time (Fig. $3 \mathrm{c}$ and Additional file 1: Figure S5). This analysis reveals that DE markers SOX17 and CXCR4 are activated surprisingly early in a small number of cells with low or no expression of $T$ (Fig. 3c and Figure S5). This trend, consistent with previous findings, confirmed the reconstructed trajectory from mesendoderm toward DE and provided a measure of confidence in the subsequent fishing step. While we only tested upregulated genes from the Wave-Crest analysis, it is possible that our top downregulated genes are playing regulatory roles for mesendoderm or mesoderm fate differentiation (Fig. 3d). Given the high quantity of reconstructed single-cell information, follow-up experiments are needed to investigate the full set of regulators governing the decision to bifurcate from mesendoderm to either mesoderm or DE.

It is not surprising that scRNA-seq data revealed cell state asynchrony during differentiation as the phenomenon has been reported in other cell types subjected to scRNA-seq analysis [26-28]. However, what factors contribute to the cell-to-cell delay or advancement of transcriptomic progression is still unclear. One possible factor is the cell cycle, which is unsynchronized across the population of cells [25] either at the initiation of differentiation and/or when single cells were collected for RNA-seq. For example, recent reports suggest that the cell cycle status can influence signaling pathways directing exit from the pluripotent state [60-62]. To resolve this, future efforts will need to monitor real-time expression of lineage specification markers while simultaneously tracing cell division.

Two new statistical tools to characterize our temporal scRNA-seq data are reported in this study. The first is SCPattern, an empirical Bayes model to identify genes with expression changes over time, specifically designed to tackle the analytical challenges of scRNA-seq data. The statistical power and simulations of SCPattern are detailed in a companion study [29]. The second tool, Wave-Crest, is composed of a first step to reconstruct temporal cell order and a second step focused on identifying novel regulators (fishing) (see "Methods"). Evaluating the performance of Wave-Crest by simulation studies can be found in Additional file 1: Supplementary
Results and Figure S8 with Additional file 7. Wave-Crest attempts to reorder cells along their differentiation progression based on known markers expression and focused on identifying novel regulators at transition state (particularly from mesendoderm to DE). Wave-Crest also uses known temporal information to improve its performance. While the development of Wave-Crest was not intended to perform pseudo-temporal reconstruction, its reconstructed single-cell order could represent a particular differentiation trajectory guided by preselected markers. While comparing the performance between Wave-Crest and other pseudo-temporal approaches is beyond the scope of this study, a preliminary comparison of using known markers to guide single-cell reordering between Wave-Crest and Monocle [63] could be found in Additional file 1: Supplementary Results and Figure S9. It is important to note that this comparison only used our preselected markers. A graphical user interface implementation of Wave-Crest is also available; details may be found in Additional file 1: Supplementary Methods and Figure S10. To decide candidate genes to use for reordering, the algorithm currently utilizes differential expression results from SCPattern combined with prior knowledge of markers (Additional file 1: Figure S5). Although supervised or semi-supervised learning plays a role in most of the single-cell analyses [27, 64-66], it would be ideal to extract markers from high dimensional data in an unsupervised fashion. The fishing step is performed primarily for transcriptional regulators in this study. It will be of great interest to perform fishing for other categories of gene families, such as posttranslational regulators or regulatory RNA species shown to be critical for cell fate transitions. Finally, control of cell-to-cell variabilities by spike-ins was not applied in our scRNA-seq data due to technical challenges in our initial in-house trial experiments. Future efforts to employ novel computational approaches to access scRNA-seq variability should greatly improve our ability to identify additional novel regulators [30, 32, 50, 66-68].

\section{$T-2 A-E G F P$ reporter line as a robust platform to define the role of $K L F 8$ in mediating DE differentiation}

In order to directly test the candidate genes identified from scRNA-seq analysis, we generated the first $T$-knockin reporter human ES cell line that faithfully reflects the endogenous $T$ protein expression level, rather than at the transcriptional level $[69,70]$. As $T$ activation marks the onset of gastrulation, this reporter line will be useful for future studies involving the earliest molecular events during the formation of the primitive streak state in human pluripotent stem cells. While CXCR4 antibody staining provided a robust readout for DE state, combining our $T$ $2 A-E G F P$ reporter with other genetically tagged reporters 
of transcription factor whose expression is important for DE cell fate would be beneficial.

Our siRNA screen resulted in mostly delayed differentiation phenotypes among the candidate genes (top upregulated genes) tested (Fig. 5b), consistent with the hypothesis that this group of genes is mostly positive regulators of the DE fate. Specifically, TERF1, SPEN, and $K L F 8$ stand out as the strongest candidates to reduce the percentage of $\mathrm{CXCR}^{+}$cells (Fig. 5b and Additional file 1: Figure S7). We concentrated on KLF8 because it displayed a dynamic expression pattern over the course of four days of differentiation (Additional file 1: Figure S7). The knockdown of TERF1 and SPEN are likely reflecting a pleiotropic rather than specific effect on DE differentiation. Interestingly, our data also indicate that KLF8 could negatively regulate $T$ expression since $K L F 8$ knockdown increased $T$ expression (Fig. 5a) and KLF8 overexpression decreased $T$ expression (Fig. 5c). Whether $T$ is a direct target of $K L F 8$ requires further examination (Fig. 6). The positive regulation role of KLF8 in DE differentiation is best demonstrated by overexpression of KLF8 alone (which is sufficient to induce DE markers) suggesting an acceleration of the transition from mesendoderm to $\mathrm{DE}$ (Figs. 5d and 6).

While the exact role(s) of $K L F 8$ during early embryogenesis has not been examined in detail, a hemizygous genetrapped $K L F 8$ allele exhibits developmental delay at midgestation; although with variable penetrance in the genetrapped embryos examined [71]. Furthermore, recent studies indicate that KLF8 mediates a number of oncogenic processes including transformation and metastasis in ovarian, breast, bladder, and colorectal cancers [72-74]. It is plausible that KLF8 may regulate the EMT that is coupled with DE specification (Fig. 6). Future efforts may elucidate the molecular mechanisms by examining the direct targets of $K L F 8$ during DE cell fate specification.

\section{Conclusions}

In this study, we reported the analysis of scRNA-seq data from a total of 1776 single cells generated from various lineage-specific progenitors and from time course profiling along mesendoderm toward DE lineage. To our knowledge, this is one of the most complete scRNA-seq studies characterizing human ES cells and their progenies to date. We describe new algorithms for scRNA-seq analysis: Wave-Crest and SCPattern. Our scRNA-seq analyses uncovered a cohort of novel regulators potentially responsible for the transitioning phase from mesendoderm toward endodermal progenitors. We have demonstrated that $K L F 8$ plays a pivotal role in accelerating the differentiation of DE cells. Altogether, we believe that the combination of scRNA-seq analysis and genetic approaches will shed light on novel molecular mechanisms governing cell fate decisions.

\section{Methods}

\section{Cell culture and differentiation}

$\mathrm{H} 1$ and H9 human ES cells were routinely maintained at the undifferentiated state in E8 medium on Matrigel (BD Bioscience) coated tissue culture plates with daily medium feeding [34]. Human ES cells were passaged every 3-4 days with $0.5 \mathrm{mM}$ EDTA in PBS at $1: 10$ to 1:15 ratio for maintenance. H1 were differentiated according to previously established protocols $[6,17,35]$. In brief, H1 cells were individualized with Accutase (Life Technologies), washed once, and then seeded onto Matrigel coated plates at a density of $1-2.0 \times 10^{4}$ cells/ $\mathrm{cm}^{2}$ and cultured in various differentiation medium. For TBs, H1 were seeded in E7 (E8 minus FGF2) with $50 \mathrm{ng} / \mathrm{mL}$ BMP4 and SB431542 $(5 \mu \mathrm{M})$. For ECs, H1 were seeded in E8 with $5 \mathrm{ng} / \mathrm{mL}$ BMP4 and $25 \mathrm{ng} / \mathrm{mL}$ Activin A for the first two days, then switched to supplement with VEGF and WNT for three days. For DE cells, $\mathrm{H} 1$ cells were seeded in E8 with BMP4 $(5 \mathrm{ng} / \mathrm{mL})$, Activin $\mathrm{A}(25 \mathrm{ng} / \mathrm{mL})$, and CHIR99021 $(1 \mu \mathrm{M})$ for the first two days, then withdraw CHIR99021 for the remaining period of differentiation. This differentiation protocol is used in all RNA-seq, hypoxic, siRNA knockdown and $K L F 8$ overexpression experiments. For NPCs, the undifferentiated H1-SOX2-mCherry reporter line was treated with $0.5 \mathrm{mM}$ EDTA in PBS for 3-5 min and seeded in NPC differentiation medium $\left(1-2 \times 10^{4}\right.$ cells $\left./ \mathrm{cm}^{2}\right)$. The NPC differentiation medium consists of E6 (E8 minus FGF2, minus TGF 1 ), with $2.5 \mu \mathrm{g} / \mathrm{mL}$ insulin, SB431542 $(10 \mu \mathrm{M})$ and $100 \mathrm{ng} / \mathrm{mL}$ Noggin [35]. DE cells, ECs, and TBs were harvested at the end of day 5 for antibody staining and subsequent FACS sorting. Specifically, ECs were enriched from the PECAM $1^{+} / \mathrm{CD} 34^{+}$double positive sorted population; DE cells were enriched from the CXCR $4^{+}$sorted population; TBs were enriched from the $\mathrm{TROP}^{+}$sorted population; and NPCs were enriched from sorting for the Cherry + population from a SOX2Cherry $^{+}$knock-in line at the end of day 7 of differentiation. For the time course scRNA-seq experiments, no cell sorting or marker enrichment was performed prior to capture single cells. All differentiation media were changed daily. HFFs were cultured in DMEM/F12 supplemented with $10 \%$ FBS. All $1.5 \% \mathrm{O}_{2}$ hypoxia experiments were conducted in a hypoxic glove box (Coy Labs). All $5 \% \mathrm{O}_{2}$ hypoxia experiments or normoxia $20 \% \mathrm{O}_{2}$ are conducted in standard cell culture incubators with $\left[\mathrm{N}_{2}\right]$ regulators. All the cell cultures performed in our laboratory have been routinely tested as negative for mycoplasma contamination and authenticated by cytogenetic tests.

Single-cell capture and single-cell cDNA library preparation Single-cell loading, capture, and library preparations were performed using the Fluidigm $\mathrm{C} 1$ system as described 
previously [25]. 5000-8000 cells were loaded onto a medium-sized (10-17 $\mu \mathrm{m})$ C1 Single-Cell Auto Prep IFC (Fluidigm) and the cell-loading script was used according to the manufacturer's instructions. The capture efficiency was inspected using EVOS FL Auto Cell Imaging system (Life Technologies) to perform an automated area scanning of the 96 capture sites on the IFC. Empty capture sites or sites having more than one cell captured were first noted, and those samples were later excluded from further library processing for RNA-seq. Immediately after capture and imaging, reverse transcription and cDNA amplification were performed in the $\mathrm{C} 1$ system using the SMARTer PCR cDNA Synthesis Kit (Clontech) and the Advantage 2 PCR Kit (Clontech) according to the instructions in the Fluidigm user manual. Full-length, single-cell cDNA libraries were harvested the next day from the $\mathrm{C} 1$ chip and diluted to a range of $0.1-0.3 \mathrm{ng} / \mu \mathrm{L}$. Diluted single-cell cDNA libraries were fragmented and amplified using the Nextera XT DNA Sample Preparation Kit and the Nextera XT DNA Sample Preparation Index Kit (Illumina). Libraries were multiplexed at 24 or 48 libraries per lane, and single-end reads of $67 \mathrm{bp}$ were sequenced on an Illumina HiSeq 2500 system.

\section{Immunofluorescence staining and confocal image analysis}

Cells were seeded and cultured on Matrigel coated glass-bottom culture dishes (MatTek, 12- or 24-well dishes) for differentiation or treatments. Cultured cells were then washed with PBS and with BD Perm/wash buffer and then fixed with $\mathrm{BD}$ Cytofix at $4{ }^{\circ} \mathrm{C}$ for 15 min. Cells were then permeablized with $0.2 \%$ TritonX-100 (in PBS) at room temperature for $30 \mathrm{~min}$. Cells were then blocked with blocking buffer (2\% BSA and $1 \%$ FBS in PBS) for $1 \mathrm{~h}$ at room temperature followed by staining with primary antibody diluted in blocking buffer at $4{ }^{\circ} \mathrm{C}$ overnight. The next day, cells were washed three times with blocking buffer before incubated with AlexaFluor secondary antibodies (Invitrogen, 1:1000 dilutions in blocking buffer) and DAPI for $1 \mathrm{~h}$ at room temperature. Cells were then washed three times with blocking buffer and mounted on glass slides (Vectors Labs) for imaging. All the primary antibodies used in this study can be found in Additional file 8: Table S7. Immunofluorescence images were collected using a Nikon A1R laser scanning confocal microscope with Plan Apo 10x, Plan Fluor 20x Ph1 DLL, or Plan Apo 20x DIC M objectives. Images were processed using NIS Elements or ImageJ. Some z-stacks were presented as maximum intensity projection images.

\section{Gene targeting and plasmids construction} Brachyury (T)-2A-EGFP reporter T-2A-EGFP-PGK-Puro targeting vector (donor vector) with $\sim 480$ bp homology arms on each side of the stop codon was cloned into the targeting vector backbone (modified from Addgene 31938) [75]. FseI and SacI linearized T-2A-EGFP-PGK-Puro cassettes were used for gene targeting experiments. All the DNA oligos used in this study are listed in Additional file 9: Table S8. CRISPR/Cas 9 mediated gene targeting experiments were performed as previously described [55]. Briefly, H9 cells were individualized with Accutase, washed once with E8 medium, and resuspended in E8 medium with $10 \mathrm{mM}$ HEPES buffer (pH 7.2-7.5) (Life Technologies). For electroporation, $2.5 \times 10^{6}$ cell were mixed with $7.5 \mu \mathrm{g}$ of pCMV-hCas9 plasmid (Addgene 41815) [56], $7.5 \mu \mathrm{g}$ of sgRNA construct, and $10 \mu \mathrm{g}$ of linearized donor DNA template in a total of $500 \mu \mathrm{L}$ of cell/DNA suspension, transferred to a 4-mm cuvette (Bio-Rad) and electroporated with a Bio-Rad Gene Pulser Xcell. Electroporation parameters were $250 \mathrm{~V}, 500 \mu \mathrm{F}$, and infinite resistance. Cells were then plated into Matrigel-coated culture dishes in E8 medium supplied with $10 \mu \mathrm{M}$ ROCK inhibitor Y-27632 (Tocris). Medium was changed daily. Puromycin selection was started three to four days after electroporation. Puromycin-resistant colonies were picked five to seven days after drug selection was applied. In the T-2A-EGFP targeting experiments, clone 39 was verified as correctly targeted clone with normal karyotype and is used for all the subsequent experiments in this study (Additional file 1: Figure S6). For the SOX2-Cherry reporter, a Cherry-2A-Puro cassette (donor vector) with $\sim 480$ bp homology arms on each side of the stop codon was cloned into the targeting vector backbone (modified from Addgene 31939) [75]. FseI and SacI linearized SOX2Cherry-2A-Puro cassettes were used for gene targeting performed in the same manner as described above. Corrected targeted clones were confirmed by PCR and southern blot analysis. Details about the SOX2 gene targeting will be reported elsewhere (Chu et al., in preparation). All vectors and their sequences are available upon request.

\section{The Wave-Crest method}

Wave-Crest extends the nearest-insertion algorithm to recover single-cell order following the expression of preselected markers in time course scRNA-seq data. Prior to the analyses, we scaled expression within each gene to values with mean 0 and variance 1 to ensure the values across different genes are comparable. Wave-Crest reorders cells within each time point by utilizing information from its other time points. Similar to Leng et al. [25], the Wave-Crest algorithm implements an extended nearest insertion (ENI) algorithm to reorder the cells, but with a constraint that cells from different collection time are not allowed to be mixed in the recovered order. The Wave-Crest ENI starts with $\tau$ randomly selected cells, one from each time point. These $\tau$ cells are sorted by the time course order. A $\tau+1$ th cell is chosen at 
random and inserted into the series of cells. Suppose that this cell is from time point $t_{1}$, this forms two candidate orders - insert this cell between the $t_{1}-1$ th and $t_{1}$ th cell in the original order, or between $t_{1}$ th and $t_{1}+1$ th. We evaluate each order using the aggregated mean squared error (MSE) of a polynomial regression. For a given order, the polynomial regression is fitted to the rescaled expression of each gene. For each order, the aggregated MSE of a candidate gene group is defined as the summation of the MSEs among all genes of interest. The optimal order of the first $\tau+1$ cells is then selected as the one that minimizes the aggregated MSE. This process is repeated to insert the $\tau+2$ th cell and so on, until all cells are considered. A 2-opt algorithm is then applied to avoid finding local maxima and to refine the global expression profile [54]. Evaluation of the ENI algorithm and the 2-opt algorithm may be found in Additional file 1: Supplementary Results and Figure S8e. Wave-Crest also incorporates a detection step to further identify genes with a smooth profile following the reconstructed pseudo-time, which we called "fishing". This detection (fishing) step again utilizes polynomial regression. For a gene g, we reorder its rescaled expression following the recovered order, fit a polynomial curve, and calculate its gene-specific MSE (observed MSE). A permutation test is then conducted to determine the goodness of the fit by comparing the observed MSE to a large group of simulated genes. To generate a permuted gene, we randomly pick a gene from the full set of genes under consideration and permute its cell order, fit a polynomial regression as above and calculate the MSE of the permuted gene. The MSE distribution of the permuted genes is then used to make inference about the MSE distribution under the null hypothesis (no expression change associated with the reconstructed cell order). An empirical gene's permutation $p$ value is then calculated as $\frac{\text { \#permuted MSE } \leq \text { observed MSE }}{\text { \#permuted MSE }}$, where the observed MSE indicates the g's MSE based on the reconstructed order. Genes with small permutation $p$ values are considered detected. Wave-Crest is available as an $\mathrm{R}$ package, freely available at (https://github.com/lengning/ WaveCrest). The softwares are licensed under the terms of the Apache License 2.0. A graphical user interface is also provided (Additional file 1: Figure S10), which allows users with little computational background to perform the analysis.

In the DE differentiation time course analysis described in this manuscript, the ENI algorithm and 2-opt algorithm were applied on 758 cells across six time points $(0 \mathrm{~h}, 12 \mathrm{~h}, 24 \mathrm{~h}, 36 \mathrm{~h}, 72 \mathrm{~h}$, and 96 h). Genes whose median expression is less than 10 were omitted. The fishing step was applied on the 172 cells collected at $36 \mathrm{~h}$ of differentiation (following the ENI reconstructed cell order). A total of 2178 transcriptional regulators were considered in this fishing step (Additional file 5: Table S4). We defined the top genes with expression trend within $36 \mathrm{~h}$ by taking the ones with small MSE in the polynomial fitting along these 172 cells. The genes were further classified into upregulated and downregulated groups by their expression trend along the recovered order of these 172 cells. The two groups were defined by the sign of the slope coefficient in gene-specific linear fitting. The genes with positive (negative) slope coefficient were defined as up- (down-) regulated from early-36 h cells to late-36 h cells. A total of 100,000 permutations were conducted in the permutation test.

\section{Reverse transcription (RT) and qPCR analysis}

All procedures were performed as described previously [76]. Total RNAs were purified using RNeasy kits (Qiagen) with either on-column DNase treatment or genomic DNA removal columns. Between $100 \mathrm{ng}$ to $500 \mathrm{ng}$ of purified RNAs were reverse transcribed with SuperScript VILO Master mix (Life Technologies). To perform TaqMan qPCR reactions (10 $\mu \mathrm{L}$ total volumes), $1 \mu \mathrm{L}$ of the cDNA was subsequently used in each of the triplicate qPCR reactions with individual $1 \times$ TaqMan Gene Expression assays and 1× TaqMan Universal PCR Master Mix II (Life Technologies). qPCR was performed using $\mathrm{ViiA}^{\mathrm{Tw}} 7$ System and data analysis was performed using ExpressionSuite ${ }^{\mathrm{Tm}}$ (all from Life Technologies). All TaqMan Gene Expression assays are from Life Technologies and are listed in Additional file 9: Table S8.

\section{siRNA knockdown experiments}

All the siRNA are from ON-TARGETplus siRNA SMARTpool (Healthcare/Dharmacon), listed in Additional file 10: Table S9. siRNA were dissolved in $1 \times$ siRNA buffer and stored as $16 \mu \mathrm{M}$ stock in $-80{ }^{\circ} \mathrm{C}$. One hour after cells were plated for differentiation, a final concentration of $50 \mathrm{nM}$ of each siRNA pool was transfected with RNAi MAX with OPTIM-DMEM following manufacture's protocols (Life Technologies). Each gene knockdown was performed with at least two replicates of experiments. FACS analysis was performed to measure the percentages of $\mathrm{CXCR}^{+}$and $\mathrm{EGFP}^{+}$cells for each gene knockdown at $42-$ $48 \mathrm{~h}$ post differentiation. The Differentiation Score is calculated as the (\% of CXCR4 ${ }^{+}$cells) $/\left(\%\right.$ of T-2A-EGFP ${ }^{+}$cells). The score obtained from non-targeting control siRNA (as transfection control, arbitrarily set to 1), was used to normalize all the results obtained from individual genespecific siRNA transfection experiments.

\section{PiggyBac vector construction and KLF8 overexpression clones}

The cDNA of KLF8 was obtained from GeneCopoeia as Gateway Entry vectors (GC-T1091), and was subsequently 
cloned into a PiggyBac Gateway Destination vector using Gateway LR clonase (Life Technologies), placing KLF8 downstream of a DOX-inducible promoter ( $p B-T e t O-$ $K L F 8)$ as previously reported [57]. A separate PiggyBac vector encoding $p E F 1 a-r t T A-I R E S-P u r o$ cassette (DOX-responsive transactivator along with puromycin resistance gene) was co-electroporated with $p B-T e t O-K L F 8$ and $C M V$-hyPBase plasmids or hyPBase mRNA. For electroporation, $2.5 \times 10^{6}$ of H9-T-2A-EGFP reporter cells were mixed with $30.0 \mu \mathrm{g}$ of $p B-T e t O-K L F 8$ plasmid, $1.5 \mu \mathrm{g}$ of pEF1a-rtTA-IRES-Puro plasmid, and $1.5 \mu \mathrm{g}$ of CMV-hyPBase in a total of $500 \mu \mathrm{L}$ of cell/DNA suspension, transferred to a 4-mm cuvette (Bio-Rad), and electroporated with a Bio-Rad Gene Pulser Xcell. Electroporation parameters were $250 \mathrm{~V}, 500 \mu \mathrm{F}$, and infinite resistance. Cells were then plated into Matrigel-coated culture dishes in E8 medium supplied with $10 \mu \mathrm{M}$ ROCK inhibitor Y-27632 (Tocris). Medium was changed daily. Puromycin selection $(1.0 \mu \mathrm{g} / \mathrm{mL})$ was applied three days after electroporation. Puromycin-resistant colonies were picked approximately seven days after drug selection was applied. Three independent clones with the most uniformed expression of KLF8 upon DOX treatment $(2.0 \mu \mathrm{g} / \mathrm{mL})$ were selected for subsequent experiments. The vectors and their sequences are available upon request.

\section{Bulk RNA-seq library construction}

Bulk RNA samples were collected at the time when the single-cell samples were processed. Cell pellets were lysed in Buffer RLT (Qiagen) and stored in $-80{ }^{\circ} \mathrm{C}$ until RNA isolations. Total RNA of each cell type was purified using the RNeasy Kit (Qiagen). cDNA libraries were prepared and indexed with Illumina's TruSeq RNA Sample Prep Kit v2 and sequenced on Illumina's HiSeq 2500 system with 4-6 indexed samples per lane with $51 \mathrm{bp}$ single-end reads.

\section{Read processing and mapping}

Reads were mapped via Bowtie 0.12.8 [77] against the hg19 RefSeq reference ("NM_" designated genes and mitochondrial genes from the Illumina iGenomes annotation). The mapping allows up to two mismatches and up to 20 multiple hits. The expected counts and TPMs were quantified via RSEM 1.2.3 [78].

\section{Bulk data - snapshot of progenitor cell types}

A total of 19 bulk samples from seven cell types were sequenced: duplicates for DEC, NPC, and TB; triplicates for H9, EC, and HFF; and four replicates for H1. The bulk RNA-seq data were normalized by median-by-ratio normalization.

scRNA-seq data - snapshot of progenitor cell types In total, seven cell types were considered. Cells having fewer than 5000 genes with TPM >1 were removed in quality control. A total of 1018 cells passed the quality control. In more detail, 212, 162, $138,105,159,173$, and 69 cells were considered in H1, H9, DEC, EC, HFF, NPC, and TB, respectively. The scRNA-seq data were normalized by median-byratio normalization. Genes with potential ordering effect (OE) were removed prior to downstream analyses. The $\mathrm{OE}$ genes were detected using OEFinder [79]. A gene is called $\mathrm{OE}$ if it has an OEFinder $p$ value $\leq 0.01$ in at least one cell type. A total of $392 \mathrm{OE}$ genes were removed in various cell type datasets.

\section{Bulk data - time course experiment}

Triplicates were sequenced from each of the six time points $-0 \mathrm{~h}, 12 \mathrm{~h}, 24 \mathrm{~h}, 36 \mathrm{~h}, 72 \mathrm{~h}$, and $96 \mathrm{~h}$. The bulk RNA-seq data were normalized by median-by-ratio normalization.

\section{scRNA-seq data - time course experiment}

In total, six time points were considered. Cells having fewer than 5000 genes with TPM $>1$ were removed in quality control. A total of 758 cells passed the quality control. In more detail, 92, 102, 66, 172, 138, and 188 cells were considered in $0 \mathrm{~h}, 12 \mathrm{~h}, 24 \mathrm{~h}, 36 \mathrm{~h}, 72 \mathrm{~h}$, and $96 \mathrm{~h}$, respectively. The scRNA-seq data were normalized by median-by-ratio normalization. Genes with potential OE were removed prior to downstream analyses. A total of $536 \mathrm{OE}$ genes were removed in the time course dataset.

\section{Bulk-supervised PCA}

Previous publications showed that directly performing PCA on single-cell data may capture unwanted noise [80]. Therefore, bulk-supervised PCA were conducted to investigate differences between different cell types in the following manner. Denote the normalized bulk RNA-seq expression of gene $g$ in sample $s$ as $Y_{g}^{s}$. Prior to PCA analysis, for each gene $g$, the bulk RNA-seq expression values were rescaled to values with mean 0 and standard deviation 1 (denote as $\tilde{Y}_{g}^{s}$ ). Similarly, denote normalized scRNA-seq expression of gene $g$ in cell $j$ as $X_{g}^{j}$ and denote the rescaled data as $\tilde{X}_{g}^{j}$. We applied PCA on the rescaled bulk RNA-seq data and obtain loadings of PCs. Denote loading of gene $g$ in PC $n$ as $W_{g}^{n}$. The bulksupervised PCA transformed data of cell $j$ in PC $n$ is then calculated as $\sum_{g} W_{g}^{n} \tilde{X}_{g}^{j}$.

Differential expression analysis in scRNA-seq time course data by SCPattern

SCPattern was used to identify genes with expression changes in the scRNA-seq time course data. For each 
gene, SCPattern calculates the posterior probability of being each possible expression path (e.g. Up-Up-UpUp-Up, Up-Up-Up-Up-Down, etc.). A gene is called "differentially expressed" if its most likely path is not EE-EE-EE-EE-EE (EE, equal expression). A total of 3247 differentially expressed genes were identified in the scRNA-seq time course experiment listed in Additional file 4: Table S3. The development of SCPattern is detailed in a companion study [29]. Both SCPattern and its graphical interface implementation are freely available at https://github.com/lengning/SCPattern. The software is licensed under the terms of the Apache License 2.0.

\section{Additional software used in this study}

To generate figures and text, the following software packages were used: Microsoft Word, Excel, and PowerPoint for Mac v14.5.6; Adobe Illustrator CSS v15.0.2; Endnote X7.3.1; and Prism 6 for Mac v6.0e. Fluidigm SINGuLAR Analysis Toolset (fluidigmSC v.3.0.3).

\section{Additional files}

Additional file 1: Figure S1. Experimental outline and qualities of scRNA-seq on human ES-derived progenitors. Figure S2. PCA and heterogeneity analysis of human ES-derived progenitors. Figure S3. Characterizations of the impacts of hypoxia on DE differentiation. Figure S4. Quality control and PCA of time course scRNA-seq experiments. Figure S5. Genes used for Wave-Crest to reconstruct DE differentiation trajectory. Figure S6. Characterizations of T-2A-EGFP knock-in reporter. Figure S7. Summary of siRNA experiments and characterization of top candidate genes. Figure S8. Simulation results evaluating Wave-Crest. Figure S9. Monocle results on DE differentiation. Figure S10. Screenshot of Wave-Crest graphical user interface. Supplementary Results and Supplementary Methods. (PDF $2613 \mathrm{~kb}$ )

Additional file 2: Table S1. List of genes used in Fig. 1c for hierarchical clustering. (XLSX $30 \mathrm{~kb}$ )

Additional file 3: Table S2. List of the $\mathrm{GO}$ terms by Allez enrichment analysis. (XLSX $62 \mathrm{~kb}$ )

Additional file 4: Table S3. List of SCPattern identified stage-specific 3247 genes. (XLSX $167 \mathrm{~kb}$ )

Additional file 5: Table S4. List of 2178 genes used for Wave-Crest fishing step. (XLSX $54 \mathrm{~kb}$ )

Additional file 6: Table S5. List of the top 150 genes identified by Wave-Crest fishing step. (XLSX 56 kb)

Additional file 7: Table S6. List of kernel signals for Wave-Crest simulation studies. (XLSX $21 \mathrm{~kb}$ )

Additional file 8: Table S7. List of the antibodies used in this study. (XLSX $43 \mathrm{~kb}$ )

Additional file 9: Table S8. List of the DNA oligos and qPCR assays used in this study. (XLSX $32 \mathrm{~kb}$ )

Additional file 10: Table S9. List of the siRNA used in this study. (XLSX $46 \mathrm{~kb})$

\section{Abbreviations}

$D E$, definitive endoderm; DEC, definitive endoderm cell; DOX, doxycycline; EC, endothelial cell; EMT, epithelial-to-mesenchymal transition; ES, embryonic stem; FACS, fluorescence-activated cell sorting; GO, gene ontology; HFF, human foreskin fibroblast; NPC, neuronal progenitor cell; PCA, principal component analysis; scRNA-seq, single-cell RNA sequencing; TB, trophoblast-like cells

\section{Acknowledgements}

We thank C. Barry, K. Vermillion, and M. Holland for critical reading of the manuscript. We thank M. Probasco and N. Propson for their assistance of FACS sorting cells; and J. Bolin, A. Elwell, and B.K. Nguyen for the preparation and sequencing of the RNA-seq samples. We thank P. Jiang and S. Swanson for performing the RNA-seq read processing.

\section{Funding}

This work was supported by the National Institutes of Health 4UH3TR000506-03 (JAT), 5U01HL099773-06 (JAT), GM102756 (CK), U54 Al1 17924 (CK) and the Charlotte Geyer Foundation, and the Morgridge Institute for Research.

\section{Availability of data and materials}

The data discussed in this study have been deposited in NCBI's Gene Expression Omnibus and are accessible through GEO Series accession number GSE75748 (https://www.ncbi.nlm.nih.gov/geo/query/ acc.cgi?acc=GSE75748). Wave-Crest is freely available as an R package (https://github.com/lengning/WaveCrest). The software is licensed under the terms of the Apache License 2.0. Both SCPattern and its graphical interface implementation are freely available at https://github.com/lengning/SCPattern. The software is licensed under the terms of the Apache License 2.0.

\section{Authors' contributions}

L-FC and NL designed and conceptualized the study and wrote the manuscript. L-FC conducted experiments and, together with $\mathrm{NL}$, analyzed data. NL performed statistical analysis and developed algorithms for RNA-seq data. JZ and ZH performed the differentiation of EC and NPC described in Fig. 1. and also helped with scRNA-seq analysis and gene targeting experiments, DM performed qPCR experiments described in Figs. 2 and 5. DTV provided the PiggyBac constructs, hyPBase constructs and mRNA, and guidance to enable L-FC's inducible transgene experiments described in Fig. 5. JC helped with Wave-Crest implementation. CK and RMS supervised the statistical methods and experimental designs. JAT designed and supervised the study and wrote the manuscript. All authors read and approved the final manuscript.

\section{Competing interests}

The authors declare that they have no competing interests.

\section{Ethics approval and consent to participate}

The experimental procedures for $\mathrm{H} 1$ and $\mathrm{H} 9$ human $\mathrm{ES}$ cells described in this study were approved by the ethics committee with IRB Approval Number: SC-2015-0010. The H1 or H9 human ES cells are registered in the NIH Human Embryonic Stem Cell Registry with the Approval Number: NIHhESC-10-0043 (http://grants.nih.gov/stem_cells/registry/current.htm?id=29) or NIHhESC-10-0062 (http://grants.nih.gov/stem_cells/registry/current.htm?id=414), respectively.

\section{Author details}

${ }^{1}$ Morgridge Institute for Research, Madison, WI, USA. ${ }^{2}$ Department of Cell \& Regenerative Biology, University of Wisconsin-Madison, Madison, WI, USA. ${ }^{3}$ Department of Molecular, Cellular, \& Developmental Biology, University of California, Santa Barbara, CA, USA. ${ }^{4}$ Department of Statistics, University of Wisconsin, Madison, WI, USA. ${ }^{5}$ Department of Biostatistics and Medical Informatics, University of Wisconsin, Madison, WI, USA. ${ }^{6}$ Present address: Genentech, Inc., South San Francisco, CA, USA. 'Present address: Department of Cell Biology, Harvard Medical School, Boston, MA, USA.

Received: 9 April 2016 Accepted: 27 July 2016

Published online: 17 August 2016

References

1. Lewis $S L$, Tam PP. Definitive endoderm of the mouse embryo: formation, cell fates, and morphogenetic function. Dev Dyn. 2006;235:2315-29.

2. Tam PP, Loebel DA. Gene function in mouse embryogenesis: get set for gastrulation. Nat Rev Genet. 2007;8:368-81.

3. Zorn AM, Wells JM. Vertebrate endoderm development and organ formation. Annu Rev Cell Dev Biol. 2009:25:221-51.

4. Kojima Y, Tam OH, Tam PP. Timing of developmental events in the early mouse embryo. Semin Cell Dev Biol. 2014;34:65-75.

5. Thomson JA, Itskovitz-Eldor J, Shapiro SS, Waknitz MA, Swiergiel JJ, Marshall VS, et al. Embryonic stem cell lines derived from human blastocysts. Science. 1998;282:1145-7. 
6. Xu RH, Chen X, Li DS, Li R, Addicks GC, Glennon C, et al. BMP4 initiates human embryonic stem cell differentiation to trophoblast. Nat Biotechnol. 2002;20:1261-4

7. D'Amour KA, Agulnick AD, Eliazer S, Kelly OG, Kroon E, Baetge EE. Efficient differentiation of human embryonic stem cells to definitive endoderm. Nat Biotechnol. 2005;23:1534-41.

8. Xu RH, Peck RM, Li DS, Feng X, Ludwig T, Thomson JA. Basic FGF and suppression of BMP signaling sustain undifferentiated proliferation of human ES cells. Nat Methods. 2005;2:185-90.

9. Yu J, Vodyanik MA, Smuga-Otto K, Antosiewicz-Bourget J, Frane JL, Tian S, et al. Induced pluripotent stem cell lines derived from human somatic cells. Science. 2007;318:1917-20.

10. Takahashi K, Tanabe K, Ohnuki M, Narita M, Ichisaka T, Tomoda K, et al. Induction of pluripotent stem cells from adult human fibroblasts by defined factors. Cell. 2007:131:861-72

11. Sumi T, Tsuneyoshi N, Nakatsuji N, Suemori H. Defining early lineage specification of human embryonic stem cells by the orchestrated balance of canonical Wnt/beta-catenin. Activin/Nodal BMP Signal Dev. 2008;135: 2969-79.

12. Yang L, Soonpaa MH, Adler ED, Roepke TK, Kattman SJ, Kennedy M, et al. Human cardiovascular progenitor cells develop from a KDR+ embryonicstem-cell-derived population. Nature. 2008;453:524-8.

13. Yu J, Hu K, Smuga-Otto K, Tian S, Stewart R, Slukvin II, et al. Human induced pluripotent stem cells free of vector and transgene sequences. Science. 2009;324:797-801

14. Chambers SM, Fasano CA, Papapetrou EP, Tomishima M, Sadelain M, Studer L. Highly efficient neural conversion of human ES and iPS cells by dual inhibition of SMAD signaling. Nat Biotechnol. 2009;27:275-80.

15. Green MD, Chen A, Nostro MC, d'Souza SL, Schaniel C, Lemischka IR, et al. Generation of anterior foregut endoderm from human embryonic and induced pluripotent stem cells. Nat Biotechnol. 2011;29:267-72.

16. Elliott DA, Braam SR, Koutsis $\mathrm{K}, \mathrm{Ng}$ ES, Jenny $\mathrm{R}$, Lagerqvist EL, et al. NKX2-5(eGFP/w) hESCs for isolation of human cardiac progenitors and cardiomyocytes. Nat Methods. 2011:8:1037-40.

17. Xie W, Schultz MD, Lister R, Hou Z, Rajagopal N, Ray P, et al. Epigenomic analysis of multilineage differentiation of human embryonic stem cells. Cell. 2013;153:1134-48

18. Loh KM, Ang LT, Zhang J, Kumar V, Ang J, Auyeong JQ, et al. Efficient endoderm induction from human pluripotent stem cells by logically directing signals controlling lineage bifurcations. Cell Stem Cell. 2014;14:237-52.

19. Gouon-Evans V, Boussemart L, Gadue P, Nierhoff D, Koehler Cl, Kubo A, et al. BMP-4 is required for hepatic specification of mouse embryonic stem cell-derived definitive endoderm. Nat Biotechnol. 2006;24:1402-11.

20. Yu P, Pan G, Yu J, Thomson JA. FGF2 sustains NANOG and switches the outcome of BMP4-induced human embryonic stem cell differentiation. Cell Stem Cell. 2011:8:326-34.

21. Faial T, Bernardo AS, Mendjan S, Diamanti E, Ortmann D, Gentsch GE, et al. Brachyury and SMAD signalling collaboratively orchestrate distinct mesoderm and endoderm gene regulatory networks in differentiating human embryonic stem cells. Development. 2015;142:2121-35.

22. Kubo A, Shinozaki K, Shannon JM, Kouskoff V, Kennedy M, Woo S, et al. Development of definitive endoderm from embryonic stem cells in culture. Development. 2004;131:1651-62.

23. Islam S, Kjallquist U, Moliner A, Zajac P, Fan JB, Lonnerberg P, et al. Characterization of the single-cell transcriptional landscape by highly multiplex RNA-seq. Genome Res. 2011;21:1160-7.

24. Ramskold D, Luo S, Wang YC, Li R, Deng Q, Faridani OR, et al. Full-length mRNA-Seq from single-cell levels of RNA and individual circulating tumor cells. Nat Biotechnol. 2012;30:777-82.

25. Leng N, Chu LF, Barry C, Li Y, Choi J, Li X, et al. Oscope identifies oscillatory genes in unsynchronized single-cell RNA-seq experiments. Nat Methods. 2015:12:947-50

26. Shalek AK, Satija R, Shuga J, Trombetta JJ, Gennert D, Lu D, et al. Single-cell RNA-seq reveals dynamic paracrine control of cellular variation. Nature. 2014;510:363-9.

27. Trapnell C. Defining cell types and states with single-cell genomics. Genome Res. 2015;25(10):1491-8. doi:10.1101/gr.190595.115.

28. Shin J, Berg DA, Zhu Y, Shin JY, Song J, Bonaquidi MA, Enikolopov G, Nauen DW, Christian KM, Ming GL, et al. Single-cell RNA-Seq with waterfall reveals molecular cascades underlying adult neurogenesis. Cell Stem Cell. 2015;17:360-72.
29. Leng N, Chu LF, Choi J, Kendziorski C, Thomson JA, Stewart RM. SCPattern: A statistical approach to identify and classify expression changes in single cell RNA-seq experiments with ordered conditions. bioRxiv. 2016. doi:10.1101/046110

30. Brennecke P, Anders S, Kim JK, Kolodziejczyk AA, Zhang X, Proserpio V, et al. Accounting for technical noise in single-cell RNA-seq experiments. Nat Methods. 2013;10:1093-5.

31. Kim JK, Marioni JC. Inferring the kinetics of stochastic gene expression from single-cell RNA-sequencing data. Genome Biol. 2013;14:R7.

32. Islam S, Zeisel A, Joost S, La Manno G, Zajac P, Kasper M, et al. Quantitative single-cell RNA-seq with unique molecular identifiers. Nat Methods. 2014;11:163-6.

33. Stegle O, Teichmann SA, Marioni JC. Computational and analytical challenges in single-cell transcriptomics. Nat Rev Genet. 2015;16:133-45

34. Chen G, Gulbranson DR, Hou Z, Bolin JM, Ruotti V, Probasco MD, et al. Chemically defined conditions for human iPSC derivation and culture. Nat Methods. 2011:8:424-9.

35. Schwartz MP, Hou Z, Propson NE, Zhang J, Engstrom CJ, Costa VS, et al. Human pluripotent stem cell-derived neural constructs for predicting neural toxicity. Proc Natl Acad Sci U S A. 2015;112:12516-21.

36. Rodaway A, Takeda H, Koshida S, Broadbent J, Price B, Smith JC, et al. Induction of the mesendoderm in the zebrafish germ ring by yolk cell-derived TGF-beta family signals and discrimination of mesoderm and endoderm by FGF. Development. 1999;126:3067-78.

37. Tada S, Era T, Furusawa C, Sakurai H, Nishikawa S, Kinoshita M, et al. Characterization of mesendoderm: a diverging point of the definitive endoderm and mesoderm in embryonic stem cell differentiation culture. Development. 2005;132:4363-74.

38. Newton MA, Quintana FA, Den Boon JA, Sengupta S, Ahlquist P. Randomset methods identify distinct aspects of the enrichment signal in gene-set analysis. Ann Appl Stat. 2007;1:85-106.

39. Simon MC, Keith B. The role of oxygen availability in embryonic development and stem cell function. Nat Rev Mol Cell Biol. 2008:9:285-96.

40. Mohyeldin A, Garzon-Muvdi T, Quinones-Hinojosa A. Oxygen in stem cell biology: a critical component of the stem cell niche. Cell Stem Cell. 2010;7:150-61

41. McGrath KE, Koniski AD, Maltby KM, McGann JK, Palis J. Embryonic expression and function of the chemokine SDF-1 and its receptor, CXCR4. Dev Biol. 1999;213:442-56

42. Wong AP, Chin S, Xia S, Garner J, Bear CE, Rossant J. Efficient generation of functional CFTR-expressing airway epithelial cells from human pluripotent stem cells. Nat Protoc. 2015:10:363-81.

43. Pimton P, Lecht $\mathrm{S}$, Stabler CT, Johannes G, Schulman ES, Lelkes PI. Hypoxia enhances differentiation of mouse embryonic stem cells into definitive endoderm and distal lung cells. Stem Cells Dev. 2015;24:663-76.

44. Anders $S_{1}$ Huber W. Differential expression analysis for sequence count data. Genome Biol. 2010;11:R106

45. Leng N, Dawson JA, Thomson JA, Ruotti V, Rissman Al, Smits BM, et al, EBSeq: an empirical Bayes hierarchical model for inference in RNA-seq experiments. Bioinformatics. 2013;29:1035-43.

46. Robinson MD, McCarthy DJ, Smyth GK. edgeR: a Bioconductor package for differential expression analysis of digital gene expression data. Bioinformatics. 2010;26:139-40.

47. Hardcastle TJ, Kelly KA. baySeq: empirical Bayesian methods for identifying differential expression in sequence count data. BMC Bioinformatics. 2010;11:422.

48. Trapnell C, Williams BA, Pertea G, Mortazavi A, Kwan G, van Baren MJ, et al. Transcript assembly and quantification by RNA-Seq reveals unannotated transcripts and isoform switching during cell differentiation. Nat Biotechnol. 2010;28:511-5.

49. Burden CJ, Qureshi SE, Wilson SR. Error estimates for the analysis of differential expression from RNA-seq count data. Peer J. 2014;2:e576.

50. Kharchenko PV, Silberstein L, Scadden DT. Bayesian approach to single-cell differential expression analysis. Nat Methods. 2014;11:740-2.

51. Bacher R, Kendziorski C. Design and computational analysis of single-cell RNA-sequencing experiments. Genome Biol. 2016;17:63.

52. Wang P, Rodriguez RT, Wang J, Ghodasara A, Kim SK. Targeting SOX17 in human embryonic stem cells creates unique strategies for isolating and analyzing developing endoderm. Cell Stem Cell. 2011:8:335-46. 
53. Wang P, McKnight KD, Wong DJ, Rodriguez RT, Sugiyama T, Gu X, et al. A molecular signature for purified definitive endoderm guides differentiation and isolation of endoderm from mouse and human embryonic stem cells. Stem Cells Dev. 2012;21:2273-87.

54. Croes AG. A method for solving traveling-salesman problems. Oper Res. 1958:6:791-812.

55. Hou Z, Zhang Y, Propson NE, Howden SE, Chu LF, Sontheimer EJ, et al. Efficient genome engineering in human pluripotent stem cells using Cas9 from Neisseria meningitidis. Proc Natl Acad Sci U S A. 2013;110:15644-9.

56. Mali P, Yang L, Esvelt KM, Aach J, Guell M, DiCarlo JE, et al. RNA-guided human genome engineering via Cas9. Science. 2013;339:823-6.

57. Vereide DT, Vickerman V, Swanson SA, Chu LF, McIntosh BE, Thomson JA. An expandable, inducible hemangioblast state regulated by fibroblast growth factor. Stem Cell Rep. 2014;3:1043-57.

58. Tesar PJ, Chenoweth JG, Brook FA, Davies TJ, Evans EP, Mack DL, et al. New cell lines from mouse epiblast share defining features with human embryonic stem cells. Nature. 2007:448:196-9.

59. Brons IG, Smithers LE, Trotter MW. Rugg-Gunn P, Sun B, Chuva de Sousa Lopes SM, et al. Derivation of pluripotent epiblast stem cells from mammalian embryos. Nature. 2007:448:191-5.

60. Calder A, Roth-Albin I, Bhatia S, Pilquil C, Lee JH, Bhatia M, et al. Lengthened G1 phase indicates differentiation status in human embryonic stem cells. Stem Cells Dev. 2013;22:279-95.

61. Pauklin $\mathrm{S}$, Vallier $\mathrm{L}$. The cell-cycle state of stem cells determines cell fate propensity. Cell. 2013;155:135-47.

62. Singh AM, Sun Y, Li L, Zhang W, Wu T, Zhao S, et al. Cell-cycle control of bivalent epigenetic domains regulates the exit from pluripotency. Stem Cell Rep. 2015;5:323-36.

63. Trapnell C, Cacchiarelli D, Grimsby J, Pokharel P, Li S, Morse M, et al. The dynamics and regulators of cell fate decisions are revealed by pseudotemporal ordering of single cells. Nat Biotechnol. 2014;32:381-6.

64. Bendall SC, Davis KL. el-Ad DA, Tadmor MD, Simonds EF, Chen TJ, et al. Single-cell trajectory detection uncovers progression and regulatory coordination in human B cell development. Cell. 2014:157:714-25.

65. Moignard V, Woodhouse S, Haghverdi L, Lilly AJ, Tanaka Y, Wilkinson AC, et al. Decoding the regulatory network of early blood development from single-cell gene expression measurements. Nat Biotechnol. 2015;33:269-76.

66. Buettner F, Natarajan KN, Casale FP, Proserpio V, Scialdone A, Theis FJ, et al. Computational analysis of cell-to-cell heterogeneity in single-cell RNAsequencing data reveals hidden subpopulations of cells. Nat Biotechnol. 2015;33:155-60

67. Fan J, Salathia N, Liu R, Kaeser GE, Yung YC, Herman JL, et al. Characterizing transcriptional heterogeneity through pathway and gene set overdispersion analysis. Nat Methods. 2016;13:241-4.

68. McDavid A, Finak G, Gottardo R. The contribution of cell cycle to heterogeneity in single-cell RNA-seq data. Nat Biotechnol. 2016;34:591-3.

69. Kita-Matsuo H, Barcova M, Prigozhina N, Salomonis N, Wei K, Jacot JG, et al. Lentiviral vectors and protocols for creation of stable hESC lines for fluorescent tracking and drug resistance selection of cardiomyocytes. PLoS One. 2009;4:e5046

70. Mendjan S, Mascetti VL, Ortmann D, Ortiz M, Karjosukarso DW, Ng Y, et al. NANOG and CDX2 pattern distinct subtypes of human mesoderm during exit from pluripotency. Cell Stem Cell. 2014:15:310-25.

71. Cox BJ, Vollmer M, Tamplin O, Lu M, Biechele S, Gertsenstein M, et al. Phenotypic annotation of the mouse X chromosome. Genome Res. 2010;20:1154-64.

72. Liang K, Liu T, Chu N, Kang J, Zhang R, Yu Y, et al. KLF8 is required for bladder cancer cell proliferation and migration. Biotechnol Appl Biochem. 2015;62:628-33

73. Yan Q, Zhang W, Wu Y, Wu M, Zhang M, Shi X, et al. KLF8 promotes tumorigenesis, invasion and metastasis of colorectal cancer cells by transcriptional activation of FHL2. Oncotarget. 2015;6:25402-17.

74. Wang $X$, Zhao J. KLF8 transcription factor participates in oncogenic transformation. Oncogene. 2007;26:456-61.

75. Hockemeyer D, Wang H, Kiani S, Lai CS, Gao Q, Cassady JP, et al. Genetic engineering of human pluripotent cells using TALE nucleases. Nat Biotechnol. 2011:29:731-4.

76. Chu LF, Surani MA, Jaenisch R, Zwaka TP. Blimp1 expression predicts embryonic stem cell development in vitro. Curr Biol. 2011;21:1759-65.

77. Langmead B, Trapnell C, Pop M, Salzberg SL. Ultrafast and memory-efficient alignment of short DNA sequences to the human genome. Genome Biol. 2009;10:R25
78. Li B, Dewey CN. RSEM: accurate transcript quantification from RNA-Seq data with or without a reference genome. BMC Bioinformatics. 2011;12:323.

79. Leng N, Choi J, Chu LF, Thomson JA, Kendziorski C, Stewart R. OEFinder: a user interface to identify and visualize ordering effects in single-cell RNAseq data. Bioinformatics. 2016;32:1408-10.

80. Hicks SC TM, Irizarry RA. On the widespread and critical impact of systematic bias and batch effects in single-cell RNA-Seq data. bioRxiv 2015. doi:10.1101/025528

\section{Submit your next manuscript to BioMed Central and we will help you at every step:}

- We accept pre-submission inquiries

- Our selector tool helps you to find the most relevant journal

- We provide round the clock customer support

- Convenient online submission

- Thorough peer review

- Inclusion in PubMed and all major indexing services

- Maximum visibility for your research

Submit your manuscript at www.biomedcentral.com/submit

) Biomed Central 